\title{
On new erosion models of Savage-Hutter type for avalanches
}

\author{
F. Bouchut, E.D. Fernández-Nieto† \\ A. Mangeney ${ }^{\ddagger} \&$ P.-Y. Lagrée ${ }^{\S}$
}

October 22, 2007

\begin{abstract}
In this work we study the modeling of one-dimensional avalanche flows made of a moving layer over a static base, where the interface between the two can be time dependent. We propose a general model, obtained by looking for an approximate solution with constant velocity profile to the incompressible Euler equations. This model has an energy dissipation equation that is consistent with the depth integrated energy equation of the Euler system. It has physically relevant steady state solutions, and, for constant slope, it gives a particular exact solution to the incompressible hydrostatic Euler equations. Then, we propose a simplified model, for which the energy conservation holds only up to third-order terms. Its associated eigenvalues depend on the mass exchange velocity between the static and moving layers. We show that a simplification used in some previously proposed models gives a nonconsistent energy equation. Our models do not use, nor provide, any equation for the moving interface, thus other arguments have to be used in order to close the system. With special assumptions, and in particular small velocity, we can nevertheless obtain an equation for the evolution of the interface. Furthermore, the unknown parameters of the model proposed by Bouchaud, Cates, Ravi and Edwards (BCRE model [7]) can be derived. For the quasistationary case we compare and discuss the equation for the moving interface with Khakhar's model [13].
\end{abstract}

*Département de Mathématiques et Applications, École Normale Supérieure \& CNRS, 45 rue d'Ulm, 75230 Paris cedex 05, France (fbouchut@dma.ens.fr)

†Departamento de Matemática Aplicada I, Universidad de Sevilla. E.T.S. Arquitectura. Avda, Reina Mercedes, s/n. 41012 Sevilla, Spain (edofer@us.es)

${ }_{\ddagger}^{\ddagger}$ Département de Modélisation Physique et Numérique, IPGP, 4 pl. Jussieu, 75232 Paris cedex 05, France (mangeney@ipgp.jussieu.fr) Now at Institut for NonLinear Science, UCSD, 9500 Gilman Drive, La Jolla, CA 92093-0402, USA

§Laboratoire de Modélisation en Mécanique UMR 7607k, 4 pl. Jussieu, 75232 Paris cedex 05, France (pyl@ccr.jussieu.fr) 


\section{Introduction}

This work focuses on the mathematical modeling of erosion processes in granular flows. Granular avalanches generally involve flowing zones and zones at rest. Static particles can be put into motion by the flowing particles (i.e. erosion) whereas flowing particles may suddenly stop (i.e. deposition). This static/flowing transition is typical of granular matter. Many industrial or geophysical applications are related to this problem. For example, when granular materials are mixed for industrial purposes, the presence of static and flowing zones could significantly affect the efficiency of the mixing process. This is a major issue in particular when rotating cylinders are used to deal with mixing of cereals or pills. In geophysics, erosion processes are expected to play a key role in the dynamics of snow or rock avalanches in mountainous and volcanic context [Sovilla et al., 2006; Pudasaini and Hutter, 2007]. Mangeney et al., 2007b show that erosion processes may change drastically the dynamics of an avalanche flowing over an erodible bed. Actually, when entrainment of granular material lying on the erodible bed is effective, a decelerating avalanche could become a surge moving at constant velocity along the slope.

The theoretical description and physical understanding of these processes are still challenging open problems. In some applications, e.g. avalanches in silos or surface flows over a pile of granular material, the no-flow and flowing zones can be fairly clearly separated into a granular layer flowing over a static layer of grains. The interface between the static and the flowing material changes with time due to the exchange of grains between the static and the flowing layers.

In the numerical modeling of avalanche dynamics, the existence of mass exchange between the no-flow and flowing zones is generally not taken into account. The models used in geophysics are based on the pioneering work of Savage and Hutter [22] where the whole column of granular material is assumed to be flowing. The mass and momentum equations are averaged over the depth of the granular material and a scaling analysis is performed with respect to the aspect ratio of the flowing mass, considered to be small. Under this hypothesis the avalanche is modeled by a Saint Venant type system derived in a reference frame linked to an inclined plane. The unknowns are the thickness and the mean (depth-averaged) velocity of the flowing mass. A constitutive relation based on Mohr-Coulomb plasticity theory is imposed, making it possible to relate the normal stresses through a coefficient involving the so-called basal and internal friction angles and to deduce a friction term at the base of the flow. The resulting system, derived for granular flows over an inclined plane, will be denoted here as the S-H model (for Savage-Hutter). Wieland et al. [24] generalized the S-H model for granular flows over surfaces with small lateral curvature (Pudasaini and Hutter [21]). More recently Bouchut et al. proposed two more general one-dimensional Savage-Hutter models [5]. The first one is valid for small variations of the local slope angle and makes it possible to derive an exact energy equation. The second model is developed for general slopes. In [6], Bouchut and Westdickenberg generalize the previous models for small or for general slope 
variation in two dimensions.

The S-H model has been successfully applied to simulate a wide range of laboratory experiments [21], as complex as well as self-channeling flows and the leveechannel morphology of their deposit [19]. However when applied to the collapse of granular columns [18], several limitations of the model have been detected. Actually, experimental results and discrete element simulations show that a static/flowing interface appears and changes with time during the collapse. Taking into account this static/flowing transition in a depth-averaged model requires the determination of an equation for the evolution of the moving interface. In other words, one has to specify the entrainment/deposition rate, which is the velocity perpendicular to the interface of mass that changes from the static to the flowing side or vice versa of the interface. Its parametrization is insufficiently known owing to the currently unknown appropriate physical or mechanical processes that govern the static/flowing transition. In order to avoid prescribing the evolution of the interface by ad-hoc physical or mechanical considerations, several attempts have been made to close the equations by imposing a given profile of the horizontal velocity and/or a given velocity at the static/flowing interface.

The purpose of this work is to derive mathematically accurate S-H type models for a one-dimensional flow of a layer made of a granular material moving over a static layer made of the same material. The closure relation making it possible to derive an equation for the static/flowing interface will be discussed and compared with different previously proposed models ([7], [8], [2], [13], [12]). In particular, the existence of an energy equation for the different systems will be investigated.

The proposed models will be discussed using the following notation (see Figure 1 ). The horizontal coordinate is denoted by $x$ and time by $t$. By $b(t, x)$ we denote the vertical thickness of the static layer (i. e. the position of the static/flowing interface), by $h(t, x)$ the thickness of the moving layer, measured perpendicularly to the unknown interface, and by $\theta(t, x)$ the angle between the horizontal and the tangent vector to the interface, according to Figure 1 (indeed, $\tan \theta=\partial_{x} b$ ). The variable $Z$ is a coordinate normal to the interface, attached to a point $\vec{X}$ in the moving layer.

One of the first models devoted to this erosion problem, proposed by Bouchaud, Cates, Ravi and Edwards (BCRE model, see [7], [8]), is simply based on the principle of mass conservation for density preserving materials. The variation of $h$ is driven by an advection equation with a volume exchange $\mathcal{E}(t, x)$ between the static and the flowing layer, while the time variation of $b$ compensates the volume exchange, and is equal to $-\mathcal{E}$. The volume exchange $\mathcal{E}(t, x)$ and the horizontal velocity component of the moving grains, $\mathcal{V}_{d}$, are both parameters of the model,

$$
\left\{\begin{array}{l}
\partial_{t}\left(\frac{h}{\cos \theta}\right)+\partial_{x}\left(h \mathcal{V}_{d}\right)=\mathcal{E}(t, x), \\
\partial_{t} b=-\mathcal{E}(t, x)
\end{array}\right.
$$



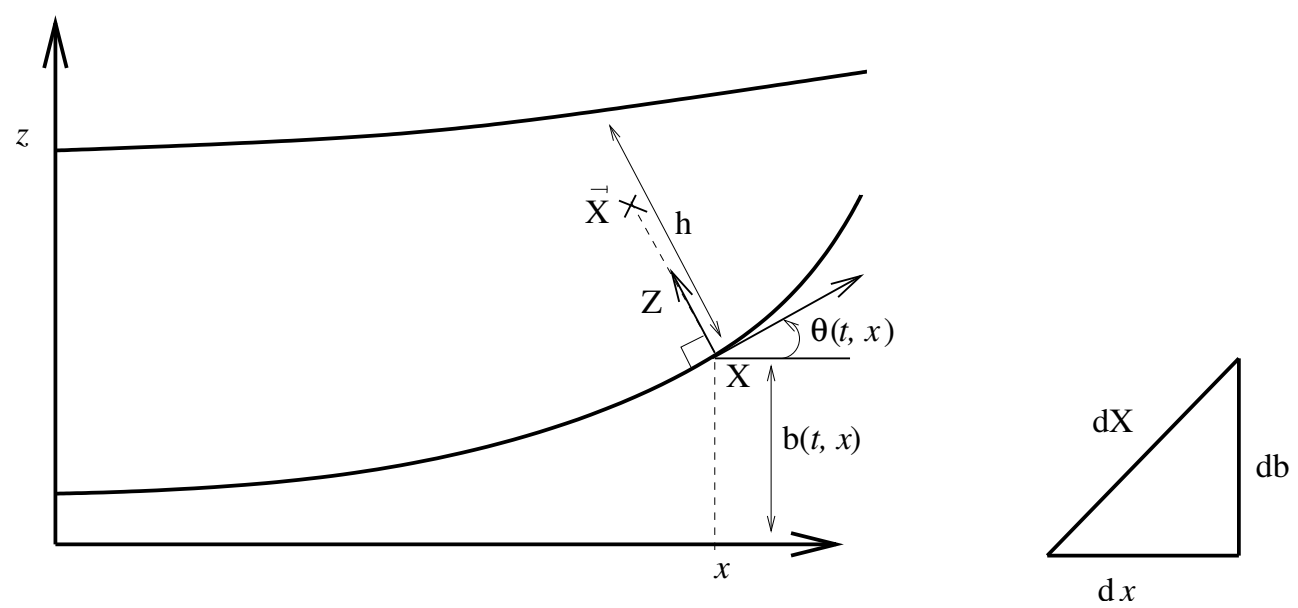

Figure 1: Interface between the static and the moving layers, and change of variable

Physically, $\mathcal{E}(t, x)$ is a volume exchange per unit time and unit horizontal line element. Although the BCRE model does not ensure conservation of momentum, it has been applied to a lot of practical problems. A difficulty of this model is to adjust the parameters $\mathcal{E}(t, x)$ and $\mathcal{V}_{d}$. Phenomenological relations have been proposed for $\mathcal{E}(t, x)$, defined as a function of the angle $\theta$ minus a neutral angle $\theta_{n}$ (if $\theta>\theta_{n}$ then erosion occurs, deposition otherwise), see [7]. The exchange term reads

$$
\mathcal{E}(t, x)=\gamma h\left(\theta-\theta_{n}\right)
$$

where $\gamma$ is an empirical constant (see also [1], [2]). The model proposed by Boutreux, Raphaël and DeGennes (BRDG model, see [8]) differs in the form of the exchange term, where $h$ is replaced by a thickness $\lambda$ smaller than $h$,

$$
\mathcal{E}(t, x)=\gamma \lambda\left(\theta-\theta_{n}\right)
$$

The idea is that only a very thin layer, of the order of the grain diameter, is involved in the exchange between the static and moving layers. Both expressions (1.2) and (1.3) can be written as

$$
\mathcal{E}(t, x)=\mathcal{V}_{u p}\left(\theta-\theta_{n}\right),
$$

where $\mathcal{V}_{u p}$ is a parameter of the model that has the dimension of a velocity.

In [2] the BCRE and BRDG models are derived from the Saint-Venant equations, making it possible to deduce the parameters involved in these models when the velocity profile is assumed to be linear with a constant slope. From this assumption, an equation for the change in time of $b$ and $h$ can be deduced. Concretely, $\mathcal{V}_{d}$ is simply obtained from the mass conservation equation when a velocity profile is assumed and $\mathcal{V}_{u p}$ is shown to depend on the specific velocity profile when a quasistationary assumption is made $\left(\theta \approx \theta_{n}\right)$. Khakhar et al. in [13], [14] derive a model based on the Saint Venant equations by also assuming a linear velocity profile. The 
equations are closed by assuming continuity of the shear stress at the static/flowing interface (this is only in conformity with the jump conditions of momentum, if $\mathcal{E} \equiv 0)$. Based on experimental data, the friction term in the flowing layer at the interface is written as the sum of a Coulomb term plus a Bagnold term whereas the friction is assumed to reduce to the Coulomb friction in the static layer at the same interface. From the equality of both expressions the value of the slope of the velocity profile can be deduced,

$$
U=U_{1} Z \quad \text { with } \quad U_{1}=f\left(\theta, \theta_{s}, \theta_{m}\right)
$$

where $\theta_{s}$ is the static angle of repose and $\theta_{m}$ the "maximum angle of repose" (see $[13])$.

Note that in [13] and [2], the pressure term $\partial_{x}\left(g h^{2} / 2\right)$ arising in the Saint Venant systems has been neglected.

In [12], Gray deduces a S-H type model for the erosion problem in avalanches in the case of rotating drums. He uses similar arguments as in the original S-H model, but by depth-averaging the equation only within the moving layer. The system of coordinates is fixed over an inclined plane with a fixed angle. For the kinematic condition he supposes that the particles on the surface that separates the moving and the static layers move with a velocity which is the sum of the velocity of the particles in the moving layer and an exchange velocity $\left(d^{b}\right)$ in the normal direction to the surface. From this kinematic condition he deduces the exchange velocity $d^{b}$ for granular flows in rotating drums. For avalanches over an inclined plane one has $d^{b}=\cos \theta \partial_{t} b$. After depth integration, a source term depending on $d^{b}$ appears in the mass balance equation. Moreover, a term proportional to $U_{b_{b}} \partial_{t} b$ arises in the momentum equation, where $U_{\left.\right|_{b}}$ is the velocity of the grains in the moving layer at the static/flowing interface. This term corresponds to the impulse of the entrained mass that must instantly assume the velocity of the moving avalanche at the interface.

In [13] the authors suppose that the velocity profile is linear with the velocity equal to zero at the static/moving interface, i.e. $U_{\left.\right|_{b}}=0$. Instead, by referring to experimental data, Gray in [12] sets $U_{\left.\right|_{b}}=u$, the averaged velocity over the moving layer. The question is whether these closure arguments, in particular using the velocity profile and/or the velocity $U_{l_{b}}$, are justified. A possible way to address this problem is to look for an approximate solution to the Euler equations that satisfies the required conditions. When simple solutions cannot be calculated, another criterion is to check that the assumptions lead to a system of equations which exhibits good mathematical properties, i.e. to have the right steady states and to satisfy an energy dissipation inequality. The main model that we propose, (3.8)-(3.9) or its simplified version (3.20)-(3.21), is coherent with these two criteria and is based on the assumption of a uniform velocity profile. We have not been able to offer such a justification for a model that is based on the assumption of a linear profile of the velocity. In Subsection 3.6 we nevertheless discuss the energy equation obtained for the different models by introducing a coefficient $\delta$ in front of the term $u \partial_{t} b$ that appears in the momentum equation in conservative form. The value of 
this coefficient depends on the assumptions related to the velocity profile and/or the velocity at the static/flowing interface. The associated energy equation is shown to be fully energy conservative (i.e. has a neutral balance independently of $b(t, x)$ ) only if $\delta=1 / 2$. However, the only value that we have been able to justify in terms of mathematical properties is $\delta=1$. Moreover, we show that if the term $u \partial_{t} b$ in the momentum equation is neglected (i.e. $\delta=0$ ), as is for most models proposed in the literature, the associated energy equation is not consistent with the depth-averaged energy equation of the Euler equations.

In order to close depth-averaged avalanche models, the "partial fluidization model" proposed in [3] could be a good candidate avoiding the difficulties encountered in the methods discussed above. Actually, Aranson and Tsimring present a theory based on the introduction of an order parameter $\rho$ that describes the transition between the static and the moving phases without any particular assumption on the velocity profile and/or the velocity at the static/flowing interface. This parameter is introduced in the hydrodynamic equation and a coupled equation for $\rho$ is proposed following the theory of phase transition of Ginzburg and Landau, as presented by Landau and Lifshitz in [16]. Based on this theory, Mangeney et al. in [20] succesfully simulate laboratory experiments of granular flows over erodible bed.

The remainder of the paper is organized as follows. In Section 2 we perform a change of variables, starting from the incompressible Euler equations over a moving bottom. Actually, two variable transformations are needed in order to avoid the difficulty that the variation in time of the local coordinates is not known. Moreover, the mass and energy equations associated to the Euler system are written down. In Section 3 we show how an approximate solution to the Euler system can be built in new coordinates, by assuming a constant velocity profile. We deduce a new model that satisfies a conservative energy inequality without any error term, in the sense that for regular solutions the energy equation is exactly obtained by a combination of the equations of the model. Moreover, it gives the expected steadystates corresponding to zero velocity (material at rest). For constant slope, the model gives an exact solution to the hydrostatic Euler equations. Friction terms can also be introduced in the model. An interesting observation is that this new model contains first-order terms that do not appear in previously proposed models. Then, we introduce a simplified model which is energy conservative only up to thirdorder terms in the aspect ratio. Its associated characteristic velocities depend on the exchange velocity between the layers $\partial_{t} b$. Finally, the developed models are compared to previously considered models in Section 4. The arguments proposed in the former studies are used in our model to derive an evolution equation for the interface $b$. In particular, we show that the parameters of the BCRE model found by [2] through the derivation of the Saint-Venant equations change by a factor $3 / 2$ if the term $U_{l_{b}} \partial_{t} b$, usually neglected in the momentum equation, is taken into account. 


\section{Change of variables}

In this section we perform a change of variables from Cartesian coordinates to the coordinates related to the interface. This enables us to reformulate the incompressible Euler equations in a more suitable form. We also reformulate the energy equation associated to the Euler equations in order to be able to compare the integrated energy with the energy associated to any integrated model.

We consider a one-dimensional flow comprising a moving layer over a static one, with a time-dependent interface between them. Time is denoted by $t$, the horizontal coordinate is denoted by $x, b(t, x)$ is the vertical thickness of the static layer, $h(t, x)$ is the thickness of the moving layer, measured perpendicularly to the unknown interface. The angle between the horizontal and the tangent vector to the interface is denoted by $\theta(t, x)$, and we notice that

$$
\tan \theta=\partial_{x} b
$$

Let $\vec{X}$ be an arbitrary point in the avalanche domain bounded by the basal and free surfaces. We consider the coordinate $Z$ that measures the position of $\vec{X}$ inside the moving layer, in the direction normal to the interface, as displayed on Figure 1. Thus,

$$
0<Z<h(t, x)
$$

Then the relations between the Cartesian coordinates $(t, \vec{X})$ and the coordinates $(t, x, Z)$ related to the interface is

$$
\vec{X}=(x-Z \sin \theta(t, x), b(t, x)+Z \cos \theta(t, x)) .
$$

Notice that $(x, b(t, x))$ is a point on the interface. We consider also a curvilinear variable $X(t, x)$ measuring the arc length along the interface. Thus at fixed $t$, the mapping $x \mapsto X$ is a change of variable with

$$
\partial_{x} X=\frac{1}{\cos \theta(t, x)}
$$

which, together with (2.1) yields the relation (at fixed $t)(d b / d x)^{2}+1=(d X / d x)^{2}$, as illustrated in Figure 1. However, since there are several ways to choose the origin on the curve, that could make different dependencies with respect to time, we cannot say a priori what is the value of $\partial_{t} X$. Nevertheless, from (2.1) we get

$$
\partial_{t} \theta=\cos ^{2} \theta \partial_{t x}^{2} b,\left.\quad \partial_{X} b\right|_{t}=\sin \theta
$$

where the notation $\left.\right|_{t}$ means that the derivative is taken at fixed $t$. 


\subsection{Transformation of the incompressible Euler equations}

We denote the velocity field of the moving layer by $\vec{U}$. Then the Euler equations for the moving layer can be written in Cartesian coordinates $(t, \vec{X})$ as

$$
\begin{gathered}
\nabla_{\vec{X}} \cdot \vec{U}=0, \\
\widehat{\partial}_{t} \vec{U}+\vec{U} \cdot \nabla_{\vec{X}} \vec{U}+\nabla_{\vec{X}} \cdot P=-\nabla_{\vec{X}}(\vec{g} \cdot \vec{X}),
\end{gathered}
$$

where $\widehat{\partial}_{t}=\left.\partial_{t}\right|_{\vec{X}}, \vec{g}=(0, g)$ and $g$ is the gravity constant. By $P$ we denote the pressure tensor, the negative of the stress tensor, divided by density,

$$
P=\left(\begin{array}{ll}
p_{x x} & p_{x z} \\
p_{z x} & p_{z z}
\end{array}\right),
$$

with $p_{x z}=p_{z x}$. Here we allow a full matrix, but later on we shall consider only the "true Euler" case where $P$ is isotropic, e.g. $P$ is a scalar times the identity tensor. This system is completed with a kinematic law for the evolving free surface,

the free surface is advected by the material velocity $\vec{U}$,

and with dynamic boundary conditions. At the free surface we just set

$$
P \vec{\nu}=0 \text { at } Z=h(t, x),
$$

where $\vec{\nu}$ is a unit vector normal to the free surface.

For the bottom interface, the incompressibility of the fluid comes into play, and we have to give precise assumptions on the fluid density, that we have not introduced yet. We assume that the moving part of the fluid is incompressible with uniform density $\rho_{m}$, while the part of the fluid that is at rest is also incompressible, with uniform density $\rho_{r}$. Having different densities $\rho_{m}$ and $\rho_{r}$, the model allows more generality, and it is physically more relevant, since experimental observations indicate that $\rho_{r}>\rho_{m}$. The physical interpretation of these assumptions is the following. On the one hand, when $\rho_{m}=\rho_{r}$, the whole fluid (comprising the moving part and the part at rest) is incompressible with uniform density. On the other hand, when $\rho_{r}>\rho_{m}$, the fluid that is deposited (e.g. stops moving and stays at rest) has to increase its density from $\rho_{m}$ to $\rho_{r}$, thus is being compressed through the interface; while the fluid that is eroded (e.g. starts moving), has to diminish its density from $\rho_{r}$ to $\rho_{m}$, thus is being decompressed through the interface.

The dynamic condition at the interface corresponding to this situation with two densities $\rho_{m}, \rho_{r}$ is deduced from the mass conservation, that can be written

$$
\widehat{\partial}_{t} \rho+\nabla_{\vec{X}} \cdot(\rho \vec{U})=0
$$

where $\rho$ takes the value $\rho_{m}$ in the moving part of the fluid, and $\rho_{r}$ in the part at rest. The velocity $\vec{U}$ is set to 0 in the part at rest. Writing down the Rankine-Hugoniot condition through the interface gives the boundary condition

$$
([\rho],[\rho \vec{U}]) \cdot \vec{N}=0
$$


where [...] denotes the jump of a quantity through the interface, and $\vec{N}$ denotes a time-space normal to the interface. Since the interface is defined by $z-b(t, x)=0$, one can take $\vec{N}=\left(-\partial_{t} b,-\partial_{x} b, 1\right)=\left(-\cos \theta \partial_{t} b,-\sin \theta, \cos \theta\right) / \cos \theta$. Denoting by $\vec{n}=(-\sin \theta, \cos \theta)$ the space unit vector normal to the interface, oriented upwards, the interface dynamic boundary condition (2.12) can be written

$$
\rho_{m} \vec{U} \cdot \vec{n}=-\left(\rho_{r}-\rho_{m}\right) \cos \theta \partial_{t} b \quad \text { at } Z=0
$$

For completeness, let us mention that from (2.6)-(2.7) one classically deduces the energy equation, valid if $P$ is isotropic, e.g. $P$ is a scalar times the identity tensor,

$$
\widehat{\partial}_{t}\left(\frac{|\vec{U}|^{2}}{2}+\vec{g} \cdot \vec{X}\right)+\nabla_{\vec{X}} \cdot\left(\frac{|\vec{U}|^{2}}{2} \vec{U}+P \vec{U}+(\vec{g} \cdot \vec{X}) \vec{U}\right)=0 .
$$

In what follows we perform a change of variables from Cartesian coordinates $(t, \vec{X})$ to coordinates attached to the interface, first to the variables $(t, X, Z)$, and then to the variables $(t, x, Z)$. The velocity components in the frame associated to the interface will be denoted by $(U, W)$,

$$
\left(\begin{array}{c}
U \\
W
\end{array}\right)=\left(\begin{array}{cc}
\cos \theta & \sin \theta \\
-\sin \theta & \cos \theta
\end{array}\right) \vec{U}
$$

The new stress tensor is

$$
\mathcal{P}=\left(\begin{array}{cc}
\cos \theta & \sin \theta \\
-\sin \theta & \cos \theta
\end{array}\right) P\left(\begin{array}{cc}
\cos \theta & -\sin \theta \\
\sin \theta & \cos \theta
\end{array}\right)=\left(\begin{array}{cc}
\mathcal{P}_{X X} & \mathcal{P}_{X Z} \\
\mathcal{P}_{Z X} & \mathcal{P}_{Z Z}
\end{array}\right) .
$$

We observe that, as $p_{x z}=p_{z x}$, then $\mathcal{P}_{X Z}=\mathcal{P}_{Z X}$.

Let us consider first the variables $(t, X, Z)$. In order to avoid confusion in differentiations, we shall denote the time in these variables by $\tau$, so that $\partial / \partial \tau$ means that we differentiate with respect to time at $X$ and $Z$ fixed. We first compute the Jacobian matrix of the transformation $\nabla_{\tau, X, Z}(t, \vec{X})$. According to $(2.3),(2.4)$ and (2.5) we have

$$
\nabla_{X, Z} \vec{X}=\left(\begin{array}{cc}
J \cos \theta & -\sin \theta \\
J \sin \theta & \cos \theta
\end{array}\right), \quad J=1-Z \partial_{X} \theta=\operatorname{det}\left(\nabla_{X, Z} \vec{X}\right) .
$$

Thus,

$$
\nabla_{\vec{X}}(X, Z)=\left(\nabla_{X, Z} \vec{X}\right)^{-1}=\frac{1}{J}\left(\begin{array}{cc}
\cos \theta & \sin \theta \\
-J \sin \theta & J \cos \theta
\end{array}\right)
$$

Then

$$
\nabla_{\tau, X, Z}(t, \vec{X})=\left(\begin{array}{cc}
1 & 0 \\
B & \nabla_{X, Z} \vec{X}
\end{array}\right), \quad B=\partial_{\tau} \vec{X}
$$

and by inversion

$$
\nabla_{t, \vec{X}}(\tau, X, Z)=\left(\begin{array}{cc}
1 & 0 \\
-\left(\nabla_{X, Z} \vec{X}\right)^{-1} B & \left(\nabla_{X, Z} \vec{X}\right)^{-1}
\end{array}\right) .
$$


We deduce from (2.19) that

$$
\operatorname{det}\left(\nabla_{\tau, X, Z}(t, \vec{X})\right)=\operatorname{det}\left(\nabla_{X, Z} \vec{X}\right)=J
$$

and from (2.20) that

$$
\widehat{\partial}_{t}(X, Z)=-\left(\nabla_{X, Z} \vec{X}\right)^{-1} B .
$$

In order to go to the variables $(t, x, Z)$ we shall need the Jacobian matrix between $(\tau, X)$ and $(t, x)$, thus we compute

$$
\nabla_{t, x}(\tau, X)=\left(\begin{array}{cc}
1 & 0 \\
\partial_{t} X & 1 / \cos \theta
\end{array}\right), \quad \nabla_{\tau, X}(t, x)=\left(\begin{array}{cc}
1 & 0 \\
-\cos \theta \partial_{t} X & \cos \theta
\end{array}\right) .
$$

Therefore we deduce the following rule, for any function $f(t, x, Z)$,

$$
\partial_{\tau} f=\partial_{t} f-\cos \theta\left(\partial_{t} X\right) \partial_{x} f
$$

where

$$
\left.\partial_{t} \equiv \partial_{t}\right|_{x, Z},\left.\quad \widehat{\partial}_{t} \equiv \partial_{t}\right|_{\vec{X}},\left.\quad \partial_{\tau} \equiv \partial_{t}\right|_{X, Z}
$$

Notice that this definition for $\partial_{t}$ is coherent with the previous notations whenever $f$ only depends on $t$ and $x$, and also that $\cos \theta \partial_{x} f=\partial_{X} f$. With the rule (2.24), we can deduce a formula for $B=\partial_{\tau} \vec{X}$. Using (2.3) and (2.1), we get

$$
B=\left(\begin{array}{c}
-\cos \theta \partial_{t} X \\
\partial_{t} b-\sin \theta \partial_{t} X
\end{array}\right)-Z\left(\partial_{t} \theta-\left(\partial_{t} X\right) \partial_{X} \theta\right)\left(\begin{array}{c}
\cos \theta \\
\sin \theta
\end{array}\right)
$$

Using (2.5), we can also rewrite $B$ as

$$
B=\left(-Z \cos ^{2} \theta \partial_{t x}^{2} b-\partial_{t} X\left(1-Z \partial_{X} \theta\right)\right)\left(\begin{array}{c}
\cos \theta \\
\sin \theta
\end{array}\right)+\left(\begin{array}{c}
0 \\
\partial_{t} b
\end{array}\right) .
$$

With this formula we get according to (2.22) and (2.18)

$$
\begin{gathered}
\widehat{\partial}_{t} X=-J^{-1}\left(\cos \theta B_{1}+\sin \theta B_{2}\right)=J^{-1}\left(J \partial_{t} X+Z \cos ^{2} \theta \partial_{t x}^{2} b-\sin \theta \partial_{t} b\right), \\
\widehat{\partial}_{t} Z=\sin \theta B_{1}-\cos \theta B_{2}=-\cos \theta \partial_{t} b .
\end{gathered}
$$

In order to perform the change of variables in the governing equations (2.6)-(2.7), let us recall the classical divergence chain rule formula.

Lemma 2.1 (Divergence chain rule) Let $\vec{\xi} \mapsto \vec{Y}(\vec{\xi})$ be a change of variables, and define the Jacobian matrix by $\mathcal{A}^{-1}=\nabla_{\vec{\xi}} \vec{Y}$, and its Jacobian determinant by $\mathcal{J}=\operatorname{det} \mathcal{A}^{-1}$. Then for any vector field $\vec{\Phi}$ one has

$$
\mathcal{J} \nabla_{\vec{Y}} \cdot \vec{\Phi}=\nabla_{\vec{\xi}} \cdot(\mathcal{J} \mathcal{A} \vec{\Phi})
$$


We notice also that with the same notation we have for any scalar $f$

$$
\nabla_{\vec{Y}} f=\mathcal{A}^{t} \nabla_{\vec{\xi}} f
$$

where we use the notations $A^{t}$ for the transpose of a matrix $A$.

In order to be complete, let us give a simple proof of (2.30).

Proof of Lemma 2.1. Fix a point $\vec{\xi}_{0}$, and consider a small ball $\mathcal{B}$ containing $\vec{Y}\left(\vec{\xi}_{0}\right)$, and a smooth function $\varphi(\vec{Y})$ vanishing outside of $\mathcal{B}$. Then, applying usual rules of calculus such as Gauss's law without boundary term (because $\varphi$ vanishes on the boundary) and changes of variables under an integral, we get

$$
\begin{aligned}
& \int \varphi(\vec{Y}) \nabla_{\vec{Y}} \cdot \vec{\Phi} d \vec{Y} \\
& =-\int \nabla \varphi(\vec{Y}) \cdot \vec{\Phi} d \vec{Y} \\
& =-\int \nabla \varphi(\vec{Y}(\vec{\xi})) \cdot \vec{\Phi}|\mathcal{J}| d \vec{\xi} \\
& =-\int \mathcal{A}^{t} \nabla_{\vec{\xi}}(\varphi(\vec{Y}(\vec{\xi}))) \cdot \vec{\Phi}|\mathcal{J}| d \vec{\xi} \\
& =-\int \nabla_{\vec{\xi}}(\varphi(\vec{Y}(\vec{\xi}))) \cdot \mathcal{A} \vec{\Phi}|\mathcal{J}| d \vec{\xi} \\
& =\int \varphi(\vec{Y}(\vec{\xi})) \nabla_{\vec{\xi}} \cdot(\mathcal{A} \vec{\Phi}|\mathcal{J}|) d \vec{\xi} \\
& =\int \varphi(\vec{Y}) \nabla_{\vec{\xi}} \cdot(\mathcal{A} \vec{\Phi}|\mathcal{J}|) \frac{d \vec{Y}}{|\mathcal{J}|} .
\end{aligned}
$$

This identity holds for any function $\varphi(\vec{Y})$, thus

$$
\nabla_{\vec{Y}} \cdot \vec{\Phi}=\nabla_{\vec{\xi}} \cdot(\mathcal{A} \vec{\Phi}|\mathcal{J}|) \frac{1}{|\mathcal{J}|} \text { in } \mathcal{B} .
$$

Since $\mathcal{J}$ has a constant sign, this proves the claim.

\subsection{Incompressibility condition}

By applying Lemma 2.1 with $\vec{\xi}=(X, Z)$ and $\vec{Y}=\vec{X}$, we readily obtain from the incompressibility equation (2.6) and with (2.21) that

$$
\nabla_{X, Z} \cdot\left(J \nabla_{\vec{X}}(X, Z) \vec{U}\right)=J \nabla_{\vec{X}} \cdot \vec{U}=0 .
$$

But according to (2.18) and (2.15),

$$
J \nabla_{\vec{X}}(X, Z) \vec{U}=\left(\begin{array}{cc}
\cos \theta & \sin \theta \\
-J \sin \theta & J \cos \theta
\end{array}\right) \vec{U}=\left(\begin{array}{c}
U \\
J W
\end{array}\right) .
$$


We deduce that

$$
\partial_{X} U+\partial_{Z}(J W)=0
$$

Notice that we could also use Lemma 2.1 in the time and space variables, by writing

$$
\nabla_{\tau, X, Z} \cdot\left(J \nabla_{t, \vec{X}}(\tau, X, Z)(0, \vec{U})\right)=J \nabla_{t, \vec{X}} \cdot(0, \vec{U})=0,
$$

which gives (2.36) since by (2.20) and (2.18)

$$
J \nabla_{t, \vec{X}}(\tau, X, Z)=\left(\begin{array}{ccc}
J & 0 & 0 \\
J \widehat{\partial}_{t} X & \cos \theta & \sin \theta \\
J \widehat{\partial}_{t} Z & -J \sin \theta & J \cos \theta
\end{array}\right) .
$$

In the same spirit, writing down

$$
\nabla_{\tau, X, Z} \cdot\left(J \nabla_{t, \vec{X}}(\tau, X, Z)(1,0)\right)=J \nabla_{t, \vec{X}} \cdot(1,0)=0,
$$

we get the identity

$$
\partial_{\tau} J+\partial_{X}\left(J \widehat{\partial}_{t} X\right)+\partial_{Z}\left(J \widehat{\partial}_{t} Z\right)=0,
$$

where $\widehat{\partial}_{t} X$ and $\widehat{\partial}_{t} Z$ can be expressed by (2.28)-(2.29). Notice that (2.40) is an identity related to the change of variables, and does not involve the unknowns $U$, $W$. Adding (2.40) and (2.36), this yields

$$
\partial_{\tau} J+\partial_{X}\left(J \widehat{\partial}_{t} X+U\right)+\partial_{Z}\left(J \widehat{\partial}_{t} Z+J W\right)=0
$$

In the sequel we shall use either (2.36) or (2.41) as incompressibility equation. They are of course equivalent.

\subsection{Momentum equations}

In order to obtain the equations for $U$ and $W$, we multiply (2.7) on the left by the matrix

$$
\left(\begin{array}{cc}
\cos \theta & \sin \theta \\
-\sin \theta & \cos \theta
\end{array}\right)
$$

that appears in (2.15). The result for the first component, multiplied by $J$, gives an equation for $U$,

$$
\begin{aligned}
& J\left(\widehat{\partial}_{t} U+\vec{U} \cdot \nabla_{\vec{X}} U\right)+J\left(\nabla_{\vec{X}}(\vec{g} \cdot \vec{X})\right) \cdot\left(\begin{array}{c}
\cos \theta \\
\sin \theta
\end{array}\right) \\
& =J W\left(\widehat{\partial}_{t} \theta+\vec{U} \cdot \nabla_{\vec{X}} \theta\right)-J \nabla_{\vec{X}} \cdot\left(P\left(\begin{array}{c}
\cos \theta \\
\sin \theta
\end{array}\right)\right)+J\left(\nabla_{\vec{X}} \theta\right)^{t} P\left(\begin{array}{c}
-\sin \theta \\
\cos \theta
\end{array}\right) .
\end{aligned}
$$

Then, according to the incompressibility condition (2.6), we can use for any scalar function $f$ the identity $\widehat{\partial}_{t} f+\vec{U} \cdot \nabla_{\vec{X}} f=\nabla_{t, \vec{X}} \cdot(f, f \vec{U})$. Applying Lemma 2.1 with 
$\vec{\xi}=(\tau, X, Z), \vec{Y}=(t, \vec{X}), \vec{\Phi}=(f, f \vec{U})$ and using (2.38), we get for any scalar function $f$

$$
J\left(\widehat{\partial}_{t} f+\vec{U} \cdot \nabla_{\vec{X}} f\right)=J \nabla_{t, \vec{X}} \cdot(f, f \vec{U})=\nabla_{\tau, X, Z} \cdot\left(\begin{array}{c}
J f \\
J\left(\widehat{\partial}_{t} X\right) f+f U \\
J\left(\widehat{\partial}_{t} Z\right) f+J f W
\end{array}\right) .
$$

We choose successively $f=U$ and $f=\theta$ in (2.44) and insert the results in (2.43). We use also Lemma 2.1 as above, but with $\vec{\Phi}=(0, P(\cos \theta, \sin \theta))$. With the help of formula (2.31) for the two remaining terms, (2.43) yields

$$
\begin{aligned}
& \nabla_{\tau, X, Z} \cdot\left(\begin{array}{c}
J U \\
J\left(\widehat{\partial}_{t} X\right) U+U^{2} \\
J\left(\widehat{\partial}_{t} Z\right) U+J U W
\end{array}\right)+\partial_{X}(\vec{g} \cdot \vec{X}) \\
& =W \nabla_{\tau, X, Z} \cdot\left(\begin{array}{c}
J \theta \\
J\left(\widehat{\partial}_{t} X\right) \theta+\theta U \\
J\left(\widehat{\partial}_{t} Z\right) \theta+J \theta W
\end{array}\right) \\
& \left.-\nabla_{X, Z} \cdot\left(\begin{array}{cc}
1 & 0 \\
0 & J
\end{array}\right)\left(\begin{array}{cc}
\cos \theta & \sin \theta \\
-\sin \theta & \cos \theta
\end{array}\right) P\left(\begin{array}{c}
\cos \theta \\
\sin \theta
\end{array}\right)\right) \\
& +\left(\nabla_{X, Z} \theta\right)^{t}\left(\begin{array}{cc}
1 & 0 \\
0 & J
\end{array}\right)\left(\begin{array}{cc}
\cos \theta & \sin \theta \\
-\sin \theta & \cos \theta
\end{array}\right) P\left(\begin{array}{c}
-\sin \theta \\
\cos \theta
\end{array}\right) .
\end{aligned}
$$

In order to obtain the equation for $W$, we multiply the first equation of (2.7) by $-\sin \theta$, and the second one by $\cos \theta$. Multiplying the sum by $J$, we get

$$
\begin{aligned}
& J\left(\widehat{\partial}_{t} W+\vec{U} \cdot \nabla_{\vec{X}} W\right)+J\left(\nabla_{\vec{X}}(\vec{g} \cdot \vec{X})\right) \cdot\left(\begin{array}{c}
-\sin \theta \\
\cos \theta
\end{array}\right) \\
& =-J U\left(\widehat{\partial}_{t} \theta+\vec{U} \cdot \nabla_{\vec{X}} \theta\right)-J \nabla_{\vec{X}} \cdot\left(P\left(\begin{array}{c}
-\sin \theta \\
\cos \theta
\end{array}\right)\right)-J\left(\nabla_{\vec{X}} \theta\right)^{t} P\left(\begin{array}{c}
\cos \theta \\
\sin \theta
\end{array}\right) .
\end{aligned}
$$

Proceeding as above, we obtain

$$
\begin{aligned}
& \nabla_{\tau, X, Z} \cdot\left(\begin{array}{c}
J W \\
J\left(\widehat{\partial}_{t} X\right) W+W U \\
J\left(\widehat{\partial}_{t} Z\right) W+J W^{2}
\end{array}\right)+J \partial_{Z}(\vec{g} \cdot \vec{X}) \\
= & -U \nabla_{\tau, X, Z} \cdot\left(\begin{array}{c}
J \theta \\
J\left(\widehat{\partial}_{t} X\right) \theta+\theta U \\
J\left(\widehat{\partial}_{t} Z\right) \theta+J \theta W
\end{array}\right) \\
& -\nabla_{X, Z} \cdot\left(\left(\begin{array}{cc}
1 & 0 \\
0 & J
\end{array}\right)\left(\begin{array}{cc}
\cos \theta & \sin \theta \\
-\sin \theta & \cos \theta
\end{array}\right) P\left(\begin{array}{c}
-\sin \theta \\
\cos \theta
\end{array}\right)\right) \\
& -\left(\nabla_{X, Z} \theta\right)^{t}\left(\begin{array}{cc}
1 & 0 \\
0 & J
\end{array}\right)\left(\begin{array}{cc}
\cos \theta & \sin \theta \\
-\sin \theta & \cos \theta
\end{array}\right) P\left(\begin{array}{c}
\cos \theta \\
\sin \theta
\end{array}\right) .
\end{aligned}
$$


At this point, we would like to simplify somewhat the equations (2.45) and (2.47). Taking into account (2.41) and the fact that $\theta$ does not depend on $Z$, we have

$$
\begin{aligned}
Q & \equiv \partial_{\tau}(J \theta)+\partial_{X}\left(J\left(\widehat{\partial}_{t} X\right) \theta+\theta U\right)+\partial_{Z}\left(J\left(\widehat{\partial}_{t} Z\right) \theta+J \theta W\right) \\
& =J \partial_{\tau} \theta+\left(J \widehat{\partial}_{t} X+U\right) \partial_{X} \theta
\end{aligned}
$$

Then, using (2.24), (2.5), expression (2.17) of $J$ and (2.28), we get

$$
\begin{aligned}
\frac{Q}{\cos \theta} & =\frac{J}{\cos \theta} \partial_{t} \theta+\left(J\left(\widehat{\partial}_{t} X-\partial_{t} X\right)+U\right) \partial_{x} \theta \\
& =\left(1-Z \cos \theta \partial_{x} \theta\right) \cos \theta \partial_{t x}^{2} b+\left(Z \cos ^{2} \theta \partial_{t x}^{2} b-\sin \theta \partial_{t} b+U\right) \partial_{x} \theta \\
& =\partial_{x}\left(\cos \theta \partial_{t} b\right)+U \partial_{x} \theta
\end{aligned}
$$

\subsection{System of equations in $(\tau, X, Z)$}

Writing down the incompressibility equation (2.36) and the momentum equations $(2.45),(2.47)$ in the variables $(\tau, X, Z)$ gives the system

$$
\begin{gathered}
\partial_{X} U+\partial_{Z}(J W)=0 \\
\partial_{\tau}(J U)+\partial_{X}\left(\left(J \widehat{\partial}_{t} X+U\right) U\right)+\partial_{Z}\left(J\left(\widehat{\partial}_{t} Z+W\right) U\right)+\partial_{X}(g(b+Z \cos \theta)) \\
=W\left(J \partial_{\tau} \theta+\left(J \widehat{\partial}_{t} X+U\right) \partial_{X} \theta\right)-\partial_{X} \mathcal{P}_{X X}-\partial_{Z}\left(J \mathcal{P}_{Z X}\right)+\mathcal{P}_{X Z} \partial_{X} \theta \\
\partial_{\tau}(J W)+\partial_{X}\left(\left(J \widehat{\partial}_{t} X+U\right) W\right)+\partial_{Z}\left(J\left(\widehat{\partial}_{t} Z+W\right) W\right)+J \partial_{Z}(g(b+Z \cos \theta)) \\
=-U\left(J \partial_{\tau} \theta+\left(J \widehat{\partial}_{t} X+U\right) \partial_{X} \theta\right)-\partial_{X} \mathcal{P}_{X Z}-\partial_{Z}\left(J \mathcal{P}_{Z Z}\right)-\mathcal{P}_{X X} \partial_{X} \theta
\end{gathered}
$$

where $\widehat{\partial}_{t} X$ and $\widehat{\partial}_{t} Z$ can be expressed by (2.28)-(2.29). Notice that according to expression (2.17) for $J$, one has $\partial_{Z} J=-\partial_{X} \theta$, thus

$$
-\partial_{Z}\left(J \mathcal{P}_{Z Z}\right)-\mathcal{P}_{X X} \partial_{X} \theta=-J \partial_{Z} \mathcal{P}_{Z Z}+\left(\mathcal{P}_{Z Z}-\mathcal{P}_{X X}\right) \partial_{X} \theta
$$

\subsection{System of equations in $(t, x, Z)$}

We now choose the horizontal coordinate $x$. We shall see that with this choice, the unknown quantity $\partial_{t} X$ from (2.28) disappears. In order to perform the change of variable to the variables $(t, x, Z)$, we use (2.24) and the identity $\cos \theta \partial_{x}=\partial_{X}$ that comes from (2.4). Moreover, we still use the notations (2.25).

At first, since $\theta$ does not depend on $Z$, we can write the incompressibility equation (2.50) as

$$
\partial_{x} U+\partial_{Z}\left(\frac{J W}{\cos \theta}\right)=0 .
$$


This equation can also be obtained from (2.50) by using Lemma 2.1. Indeed, take $\vec{Y}=(\tau, X, Z), \vec{\xi}=(t, x, Z)$. Then, according to (2.23), the matrix $\mathcal{J A}$ in $(2.30)$ is

$$
\mathcal{J A}=\left(\begin{array}{ccc}
(\cos \theta)^{-1} & 0 & 0 \\
-\partial_{t} X & 1 & 0 \\
0 & 0 & (\cos \theta)^{-1}
\end{array}\right)
$$

Multiplying this matrix by the vector $\vec{\Phi}=(0, U, J W)$ appearing in (2.50) gives the vector $(0, U, J W / \cos \theta)$. Writing that the divergence in $(t, x, Z)$ of this vector vanishes gives (2.54). Similarly, we can transform identity (2.40) to get

$$
\partial_{t}\left(\frac{J}{\cos \theta}\right)+\partial_{x}\left(-J \partial_{t} X+J \widehat{\partial}_{t} X\right)+\partial_{Z}\left(\frac{J \widehat{\partial}_{t} Z}{\cos \theta}\right)=0
$$

or more explicitly, according to (2.28)-(2.29)

$$
\partial_{t}\left(\frac{J}{\cos \theta}\right)+\partial_{x}\left(Z \cos ^{2} \theta \partial_{t x}^{2} b-\sin \theta \partial_{t} b\right)+\partial_{Z}\left(-J \partial_{t} b\right)=0 .
$$

Indeed, (2.57) can also be checked directly by using expression (2.17) of $J$ and relation (2.1) relating $\theta$ to $b$. The identity (2.57) is true for any function $b(t, x)$, and does not involve the unknowns $U, W$. Finally, adding (2.54) and (2.57), we obtain the combined incompressibility equation

$$
\partial_{t}\left(\frac{J}{\cos \theta}\right)+\partial_{x}\left(Z \cos ^{2} \theta \partial_{t x}^{2} b-\sin \theta \partial_{t} b+U\right)+\partial_{Z}\left(\frac{J}{\cos \theta}\left(-\cos \theta \partial_{t} b+W\right)\right)=0 .
$$

Next, consider equation (2.51) for $U$. Applying again the divergence chain rule, and with the use of (2.49), it takes the form

$$
\begin{aligned}
& \partial_{t}\left(\frac{J U}{\cos \theta}\right)+\partial_{x}\left(\left(J\left(\widehat{\partial}_{t} X-\partial_{t} X\right)+U\right) U+g(b+Z \cos \theta)+\mathcal{P}_{X X}\right) \\
& +\partial_{Z}\left(\frac{J}{\cos \theta}\left(\widehat{\partial}_{t} Z+W\right) U+\frac{J \mathcal{P}_{Z X}}{\cos \theta}\right)=W\left(\partial_{x}\left(\cos \theta \partial_{t} b\right)+U \partial_{x} \theta\right)+\mathcal{P}_{X Z} \partial_{x} \theta
\end{aligned}
$$

Combining it with (2.58), we may derive its nonconservative analogue

$$
\begin{aligned}
& \frac{J}{\cos \theta} \partial_{t} U+\left(Z \cos ^{2} \theta \partial_{t x}^{2} b-\sin \theta \partial_{t} b+U\right) \partial_{x} U+J\left(\frac{W}{\cos \theta}-\partial_{t} b\right) \partial_{Z} U \\
& +\partial_{x}\left(g(b+Z \cos \theta)+\mathcal{P}_{X X}\right)+\partial_{Z}\left(\frac{J \mathcal{P}_{Z X}}{\cos \theta}\right) \\
= & W\left(\partial_{x}\left(\cos \theta \partial_{t} b\right)+U \partial_{x} \theta\right)+\mathcal{P}_{X Z} \partial_{x} \theta
\end{aligned}
$$


A similar computation for $W$, again with the aid of Lemma 2.1, transforms (2.52) into

$$
\begin{aligned}
& \partial_{t}\left(\frac{J W}{\cos \theta}\right)+\partial_{x}\left(\left(J\left(\widehat{\partial}_{t} X-\partial_{t} X\right)+U\right) W+\mathcal{P}_{X Z}\right) \\
& +\partial_{Z}\left(\frac{J}{\cos \theta}\left(\widehat{\partial}_{t} Z+W\right) W+\frac{J \mathcal{P}_{Z Z}}{\cos \theta}\right)+\frac{J}{\cos \theta} \partial_{Z}(g(b+Z \cos \theta)) \\
= & -U\left(\partial_{x}\left(\cos \theta \partial_{t} b\right)+U \partial_{x} \theta\right)-\mathcal{P}_{X X} \partial_{x} \theta .
\end{aligned}
$$

If we combine this again with (2.58) as before and use (2.53), we obtain the nonconservative equation

$$
\begin{aligned}
& \frac{J}{\cos \theta} \partial_{t} W+\left(Z \cos ^{2} \theta \partial_{t x}^{2} b-\sin \theta \partial_{t} b+U\right) \partial_{x} W+J\left(\frac{W}{\cos \theta}-\partial_{t} b\right) \partial_{Z} W \\
& +\partial_{x} \mathcal{P}_{X Z}+\frac{J}{\cos \theta} \partial_{Z}\left(g(b+Z \cos \theta)+\mathcal{P}_{Z Z}\right) \\
= & -U\left(\partial_{x}\left(\cos \theta \partial_{t} b\right)+U \partial_{x} \theta\right)+\left(\mathcal{P}_{Z Z}-\mathcal{P}_{X X}\right) \partial_{x} \theta .
\end{aligned}
$$

In summary, the governing equations, comprising all the balance laws of mass and momentum and referred to the independent variables $(t, x, Z)$ is defined: (i) in conservative form by $(2.54),(2.59),(2.61)$, and (ii), in nonconservative form by $(2.54)$, $(2.60),(2.62)$.

\subsection{Energy equation}

In what follows we consider the case where $P$ is isotropic, $P=p_{z z} I$. Then we have also $\mathcal{P}=p_{z z} I$, and for simplicity we shall denote $p_{z z}$ by $P$.

Starting from the energy equation (2.14) written in Cartesian coordinates $\vec{Y}=$ $(t, \vec{X})$, we perform the change of variables to $\vec{\xi}=(\tau, X, Z)$. Applying Lemma 2.1 with (2.38) and appropriate choices for $\vec{\Phi}$ leads to

$$
\begin{aligned}
& \partial_{\tau}\left(J\left(\frac{U^{2}+W^{2}}{2}+\vec{g} \cdot \vec{X}\right)\right)+\partial_{X}\left(\left(J \widehat{\partial}_{t} X+U\right)\left(\frac{U^{2}+W^{2}}{2}+\vec{g} \cdot \vec{X}\right)+P U\right) \\
& +\partial_{Z}\left(J\left(\widehat{\partial}_{t} Z+W\right)\left(\frac{U^{2}+W^{2}}{2}+\vec{g} \cdot \vec{X}\right)+P J W\right)=0 .
\end{aligned}
$$

Then, applying Lemma 2.1 again with $\vec{Y}=(\tau, X, Z), \vec{\xi}=(t, x, Z)$, and using (2.55), we obtain the balance equation for the energy referred to the $(t, x, Z)$ coordinates as follows,

$$
\begin{aligned}
& \partial_{t}\left(\frac{J}{\cos \theta}\left(\frac{U^{2}+W^{2}}{2}+g(b+Z \cos \theta)\right)\right) \\
+ & \partial_{x}\left(\left(Z \cos ^{2} \theta \partial_{t x}^{2} b-\sin \theta \partial_{t} b+U\right)\left(\frac{U^{2}+W^{2}}{2}+g(b+Z \cos \theta)\right)+P U\right) \\
+ & \partial_{Z}\left(\frac{J}{\cos \theta}\left(-\cos \theta \partial_{t} b+W\right)\left(\frac{U^{2}+W^{2}}{2}+g(b+Z \cos \theta)\right)+\frac{P J W}{\cos \theta}\right)=0 .
\end{aligned}
$$




\subsection{Integration and kinematic boundary condition}

In this subsection we provide formulas for quantities which are integrated with respect to $Z$. Moreover, we derive the kinematic boundary condition associated to the free surface, that gives the evolution of $h(t, x)$.

According to $(2.9)$, the free surface is advected by the material velocity $\vec{U}$. Consider a material point $\vec{X}(s)$, where $s$ is the time, that is moving with the flow, and that stays on the free surface. Then

$$
\frac{d \vec{X}}{d s}(s)=\vec{U}(s, \vec{X}(s))
$$

In the variables $(t, x, Z)$, the free surface is characterized by $Z=h(t, x)$. Thus

$$
Z(s, \vec{X}(s))=h(s, x(s))
$$

where $(s, x(s))$ are the $(t, x)$ coordinates corresponding to $(s, \vec{X}(s))$. Differentiating (2.66) with respect to $s$ gives

$$
\nabla_{t, \vec{X}} Z(s, \vec{X}(s))\left(\begin{array}{c}
1 \\
\vec{U}(s, \vec{X}(s))
\end{array}\right)=\partial_{t} h(s, x(s))+\partial_{x} h(s, x(s)) \frac{d x}{d s}(s) .
$$

We compute

$$
\begin{gathered}
\frac{d x}{d s}=\partial_{\tau} x+\left(\partial_{X} x\right) \frac{d X}{d s} \\
\frac{d X}{d s}=\nabla_{t, \vec{X}} X(s, \vec{X}(s))\left(\begin{array}{c}
1 \\
\vec{U}(s, \vec{X}(s))
\end{array}\right) .
\end{gathered}
$$

Using (2.18) and (2.23), equation (2.67) yields

$$
\widehat{\partial}_{t} Z+W=\partial_{t} h+\left(-\cos \theta \partial_{t} X+\cos \theta\left(\widehat{\partial}_{t} X+U / J\right)\right) \partial_{x} h
$$

With (2.28), (2.29) we get

$$
-\cos \theta \partial_{t} b+W=\partial_{t} h+\frac{\cos \theta}{J}\left(Z \cos ^{2} \theta \partial_{t x}^{2} b-\sin \theta \partial_{t} b+U\right) \partial_{x} h .
$$

This must hold at any point on the free surface, therefore the kinematic condition is that for any $(t, x)$,

$$
\frac{J_{\left.\right|_{h}}}{\cos \theta} \partial_{t} h+\left(h \cos ^{2} \theta \partial_{t x}^{2} b-\sin \theta \partial_{t} b+U_{\left.\right|_{h}}\right) \partial_{x} h=\frac{J_{\left.\right|_{h}}}{\cos \theta}\left(-\cos \theta \partial_{t} b+W_{\left.\right|_{h}}\right),
$$

where $\left.\right|_{h}$ means that we take the value at $Z=h(t, x)$.

Now, taking into account the boundary condition (2.13), which can be written as

$$
W_{Z=0}=-\left(\frac{\rho_{r}}{\rho_{m}}-1\right) \cos \theta \partial_{t} b
$$


integrating of the incompressibility equation (2.58) for $Z$ between 0 and $h(t, x)$, and using the kinematic condition (2.71), gives

$$
\partial_{t} \int_{0}^{h} \frac{J}{\cos \theta} d Z+\partial_{x} \int_{0}^{h}\left(Z \cos ^{2} \theta \partial_{t x}^{2} b-\sin \theta \partial_{t} b+U\right) d Z=-\frac{\rho_{r}}{\rho_{m}} \partial_{t} b .
$$

More explicitly, the mass conservation reads

$$
\partial_{t}\left(\frac{h}{\cos \theta}-\frac{h^{2}}{2} \partial_{x} \theta\right)+\partial_{x}\left(\frac{h^{2}}{2} \cos ^{2} \theta \partial_{t x}^{2} b-h \sin \theta \partial_{t} b+h \bar{U}\right)=-\frac{\rho_{r}}{\rho_{m}} \partial_{t} b
$$

where

$$
\bar{U}=\frac{1}{h} \int_{0}^{h} U d Z
$$

Notice that with known (2.58) and (2.72), the mass conservation (2.74) is equivalent to the kinematic condition (2.71).

We can proceed similarly for the energy equation. Assuming that $P$ is isotropic, we integrate the energy equation (2.64) for $Z$ between 0 and $h(t, x)$. Using the kinematic condition (2.71) and the boundary conditions (2.72) and (2.10), we obtain the depth-integrated energy equation

$$
\begin{aligned}
& \partial_{t} \int_{0}^{h} \frac{J}{\cos \theta}\left(\frac{U^{2}+W^{2}}{2}+g(b+Z \cos \theta)\right) d Z \\
& +\partial_{x} \int_{0}^{h}\left(\left(Z \cos ^{2} \theta \partial_{t x}^{2} b-\sin \theta \partial_{t} b+U\right)\left(\frac{U^{2}+W^{2}}{2}+g(b+Z \cos \theta)\right)+P U\right) d Z \\
= & -\left(\frac{1}{2} U_{Z=0}^{2}+\frac{1}{2}\left(\left(\frac{\rho_{r}}{\rho_{m}}-1\right) \cos \theta \partial_{t} b\right)^{2}+g b\right) \frac{\rho_{r}}{\rho_{m}} \partial_{t} b-\left(\frac{\rho_{r}}{\rho_{m}}-1\right) P_{Z=0} \partial_{t} b .
\end{aligned}
$$

It is not possible to obtain an explicit formula for this integrated equation, unless a particular profile of the velocity in $Z$ is assumed.

The right-hand sides in (2.74) and (2.76) deserve some comments. For the mass conservation law $(2.74)$, the term $\frac{\rho_{r}}{\rho_{m}} \partial_{t} b$ can be put on the left-hand side. Then, multiplying the result by $\rho_{m}$, what one obtains is the time variation of the mass of moving fluid plus the mass of fluid at rest, which is the total mass of the fluid. For the energy equation (2.76), one can similarly put the term $\frac{\rho_{r}}{\rho_{m}} g b \partial_{t} b=\partial_{t}\left(\frac{\rho_{r}}{\rho_{m}} g b^{2} / 2\right)$ on the left-hand side, since after multiplication by $\rho_{m}$, the term $\rho_{r} g b^{2} / 2$ represents the potential energy of the static part of the fluid. However, even when $\rho_{m}=\rho_{r}$, there remains the term $-\frac{1}{2} U_{Z=0}^{2} \partial_{t} b$ on the right-hand side, that cannot be put in conservative form. This term represents the variation of the kinetic energy attached to the displacement of the interface $b(t, x)$. When $\rho_{m} \neq \rho_{r}$, the kinetic energy at the interface is completed with the normal term $W_{Z=0}^{2}$, which is non zero, and an additional term involving $P_{Z=0}$ remains on the right-hand side, related to the mass exchanges through the interface. 


\subsection{Hydrostatic model}

Classically, the hydrostatic assumption consists in removing the normal acceleration in the momentum equation. Since $W$ is the normal velocity to the bottom interface, doing this means to remove the first line in (2.62). Therefore, the hydrostatic model, formulated with respect to the coordinates $(t, x, Z)$, comprises equations $(2.54)$, (2.60), and

$$
\begin{aligned}
& \partial_{x} \mathcal{P}_{X Z}+\frac{J}{\cos \theta} \partial_{Z}\left(g(b+Z \cos \theta)+\mathcal{P}_{Z Z}\right) \\
= & -U\left(\partial_{x}\left(\cos \theta \partial_{t} b\right)+U \partial_{x} \theta\right)+\left(\mathcal{P}_{Z Z}-\mathcal{P}_{X X}\right) \partial_{x} \theta
\end{aligned}
$$

The kinematic and boundary conditions remain unchanged for this system. If we assume that $P$ is isotropic, one can directly combine (2.54), (2.60) and (2.77) to obtain a hydrostatic energy equation. More explicitly, we can proceed as follows. We first transform the equation $\widehat{\partial}_{t}(\vec{g} \cdot \vec{X})+\nabla_{\vec{X}} \cdot 0=0$ to the $(t, x, Z)$ variables; this yields the identity

$$
\begin{aligned}
\partial_{t}\left(\frac{J}{\cos \theta} g(b+Z \cos \theta)\right)+ & \partial_{x}\left(\left(Z \cos ^{2} \theta \partial_{t x}^{2} b-\sin \theta \partial_{t} b\right) g(b+Z \cos \theta)\right) \\
& +\partial_{Z}\left(-J \partial_{t} b g(b+Z \cos \theta)\right)=0
\end{aligned}
$$

that can also be checked directly. Then, we add up (2.60) times $U$ plus (2.77) times $W$ plus (2.54) times $U^{2} / 2+g(b+Z \cos \theta)+P$ plus (2.57) times $U^{2} / 2$ plus (2.78). This gives the hydrostatic energy equation

$$
\begin{aligned}
& \partial_{t}\left(\frac{J}{\cos \theta}\left(\frac{U^{2}}{2}+g(b+Z \cos \theta)\right)\right) \\
+ & \partial_{x}\left(\left(Z \cos ^{2} \theta \partial_{t x}^{2} b-\sin \theta \partial_{t} b+U\right)\left(\frac{U^{2}}{2}+g(b+Z \cos \theta)\right)+P U\right) \\
+ & \partial_{Z}\left(\frac{J}{\cos \theta}\left(-\cos \theta \partial_{t} b+W\right)\left(\frac{U^{2}}{2}+g(b+Z \cos \theta)\right)+\frac{P J W}{\cos \theta}\right)=0,
\end{aligned}
$$

the only difference with (2.64) being that the term $W^{2} / 2$ has disappeared. Indeed, the same computation for the non-hydrostatic model gives (2.64). Therefore, for the hydrostatic model, the mass conservation still reads (2.74), while the integrated energy equation takes the form

$$
\begin{aligned}
& \partial_{t} \int_{0}^{h} \frac{J}{\cos \theta}\left(\frac{U^{2}}{2}+g(b+Z \cos \theta)\right) d Z \\
+ & \partial_{x} \int_{0}^{h}\left(\left(Z \cos ^{2} \theta \partial_{t x}^{2} b-\sin \theta \partial_{t} b+U\right)\left(\frac{U^{2}}{2}+g(b+Z \cos \theta)\right)+P U\right) d Z \\
= & -\left(\frac{1}{2} U_{Z=0}^{2}+g b\right) \frac{\rho_{r}}{\rho_{m}} \partial_{t} b-\left(\frac{\rho_{r}}{\rho_{m}}-1\right) P_{Z=0} \partial_{t} b .
\end{aligned}
$$




\section{A new erosion model for avalanches}

In this section we propose a new erosion model for avalanches, derived from our formulation (2.54), (2.60), (2.62) for the incompressible equations subject to the boundary conditions (2.72), (2.10) and the mass conservation (2.74). We first deal with the case where the stress tensor $P$ is isotropic, then in Section 3.3 non-diagonal terms are considered in order to include friction effects.

Our procedure consists, as usual, in assuming for the velocity $U(t, x, Z)$ a particular profile in $Z$. An approximate solution to the incompressible system is searched for with this particular profile, retaining only lower order terms relatively to the aspect ratio characterizing the shallowness of the moving mass. Indeed, we retain all first and second-order terms, and some third-order terms that are necessary to obtain a model that verifies exactly an energy inequality. Interestingly, the resulting model contains some first-order terms that do not appear in previously proposed models.

\subsection{Formal derivation}

In what follows we consider a moving layer over a given variable bottom interface $b(t, x)$, as described at the beginning of Section 2. Thus, according to (2.1), $\theta$ is also known. We denote by $\varepsilon$ the aspect ratio between the characteristic lengths normal and parallel to the interface, which is supposed to be a small parameter. Thus we have the shallowness assumption

$$
h=\mathcal{O}(\varepsilon) .
$$

We look for a solution with a velocity profile almost constant in $Z$,

$$
U(t, x, Z)=u(t, x)+\mathcal{O}\left(\varepsilon^{2}\right) .
$$

Moreover, we assume a small variation in space of the angle $\theta$ and a small variation in time of $b$,

$$
\partial_{x} \theta=\mathcal{O}(\epsilon), \quad \partial_{t} b=\mathcal{O}(\epsilon) .
$$

Under these hypotheses, we make the following approximations. We assume that there are no singularities in time or space; i.e. $\partial_{t}$ and $\partial_{x}$ are formally bounded operators. On the other hand, the shallowness assumption leads to $\partial_{Z}=\mathcal{O}(1 / \epsilon)$. At first, since $0<Z<h,(3.1)$ ensures that $Z=\mathcal{O}(\varepsilon)$. Notice that (2.54), together with $(2.72)$, can be used to define $W(t, x, Z)$, once $U(t, x, Z)$ is known. From this we deduce that $W=\mathcal{O}(\varepsilon), \partial_{t} W=\mathcal{O}(\varepsilon), \partial_{x} W=\mathcal{O}(\varepsilon)$. With (3.3) we have $J=$ $1+\mathcal{O}\left(\varepsilon^{2}\right)$, and $(2.62),(3.3)$ give

$$
\partial_{Z}(g Z \cos \theta+P)=\mathcal{O}(\varepsilon)
$$

implying together with (2.10) the near hydrostatic pressure

$$
P=g \cos \theta(h-Z)+\mathcal{O}\left(\varepsilon^{2}\right) .
$$


Substituting this expression into (2.60), we obtain, on using (3.2),

$\frac{1-Z \cos \theta \partial_{x} \theta}{\cos \theta} \partial_{t} u+\left(Z \cos ^{2} \theta \partial_{t x}^{2} b-\sin \theta \partial_{t} b\right) \partial_{x} u+\partial_{x}\left(\frac{u^{2}}{2}+g h \cos \theta+g b\right)=\mathcal{O}\left(\varepsilon^{2}\right)$.

We observe that the terms depending on $Z$, i.e. $Z \cos \theta \partial_{x} \theta$ and $Z \cos ^{2} \theta \partial_{t x}^{2} b$, are second-order terms, according to (3.3). Therefore, they can be neglected; thus we get an equation in the variables $(t, x)$ only. This justifies the compatibility of the choice (3.2) with the Euler system. However, although the two previously mentioned terms depending on $Z$ can be neglected, we keep them because they are necessary in order to obtain a system which verifies exactly an energy inequality as will be discussed in the following section. Indeed, in order to remove the dependence in $Z$, we can take $Z=h / 2$ in (3.6) without changing the order of approximation. We close the system by rewriting (2.74), where $\bar{U}=u+\mathcal{O}\left(\varepsilon^{2}\right)$. This gives an error in $\mathcal{O}\left(\varepsilon^{3}\right)$ in $(2.74)$. Finally, by expanding $(2.76)$ we obtain the following equation for the energy,

$$
\begin{aligned}
& \partial_{t}\left(\left(\frac{h}{\cos \theta}-\frac{h^{2}}{2} \partial_{x} \theta\right)\left(\frac{u^{2}}{2}+g b\right)+g \frac{h^{2}+\frac{\rho_{r}}{\rho_{m}} b^{2}}{2}-g \frac{h^{3}}{3} \cos \theta \partial_{x} \theta\right) \\
& +\partial_{x}\left(\left(\frac{u^{2}}{2}+g h \cos \theta+g b\right) h u+\left(\frac{h^{2}}{2} \cos ^{2} \theta \partial_{t x}^{2} b-h \sin \theta \partial_{t} b\right)\left(\frac{u^{2}}{2}+g b\right)\right. \\
& \left.+g \frac{h^{3}}{3} \cos ^{3} \theta \partial_{t x}^{2} b-g \frac{h^{2}}{2} \sin \theta \cos \theta \partial_{t} b\right) \\
& =-\frac{u^{2}}{2} \frac{\rho_{r}}{\rho_{m}} \partial_{t} b-\left(\frac{\rho_{r}}{\rho_{m}}-1\right) g h \cos \theta \partial_{t} b+\mathcal{O}\left(\varepsilon^{3}\right) .
\end{aligned}
$$

\subsection{Properties of the model}

In this subsection we study the properties of the model obtained as explained above, and dropping the error terms. The model is defined by the two mass and momentum equations coming from (2.74) and (3.6) (with $Z=h / 2)$,

$$
\begin{gathered}
\partial_{t}\left(\frac{h}{\cos \theta}-\frac{h^{2}}{2} \partial_{x} \theta\right)+\partial_{x}\left(\frac{h^{2}}{2} \cos ^{2} \theta \partial_{t x}^{2} b-h \sin \theta \partial_{t} b+h u\right)=-\frac{\rho_{r}}{\rho_{m}} \partial_{t} b \\
\left(\frac{1}{\cos \theta}-\frac{h}{2} \partial_{x} \theta\right) \partial_{t} u+\left(\frac{h}{2} \cos ^{2} \theta \partial_{t x}^{2} b-\sin \theta \partial_{t} b\right) \partial_{x} u+\partial_{x}\left(\frac{u^{2}}{2}+g h \cos \theta+g b\right)=0 .
\end{gathered}
$$

Notice that when $\partial_{t} b=0$, the system differs slightly from the model proposed in [5]. As usual, (3.9) is valid for smooth solutions, but for possibly discontinuous solutions, one should rather write the momentum equation in conservative form

$$
\begin{aligned}
& \partial_{t}\left(\left(\frac{h}{\cos \theta}-\frac{h^{2}}{2} \partial_{x} \theta\right) u\right)+\partial_{x}\left(\left(\frac{h^{2}}{2} \cos ^{2} \theta \partial_{t x}^{2} b-h \sin \theta \partial_{t} b+h u\right) u+g \frac{h^{2}}{2} \cos \theta\right) \\
& =-g \sin \theta\left(\frac{h}{\cos \theta}-\frac{h^{2}}{2} \partial_{x} \theta\right)-\frac{\rho_{r}}{\rho_{m}} u \partial_{t} b,
\end{aligned}
$$


obtained by multiplying (3.8) by $u,(3.9)$ by $h$ and adding the results. The term $g \frac{h^{2}}{2} \sin \theta \partial_{x} \theta$ on the right-hand side of (3.10) was introduced by Bouchut et al. in [5]. Here it plays the same role, to maintain a conservative energy balance and to have the lake at rest solution.

Theorem 3.1 System (3.8)-(3.9) has the following properties.

(i) It admits an energy dissipation inequality

$$
\begin{aligned}
& \partial_{t}\left(\left(\frac{h}{\cos \theta}-\frac{h^{2}}{2} \partial_{x} \theta\right)\left(\frac{u^{2}}{2}+g b\right)+g \frac{h^{2}+\frac{\rho_{r}}{\rho_{m}} b^{2}}{2}-g \frac{h^{3}}{3} \cos \theta \partial_{x} \theta\right) \\
& +\partial_{x}\left(\left(\frac{u^{2}}{2}+g h \cos \theta+g b\right) h u+\left(\frac{h^{2}}{2} \cos ^{2} \theta \partial_{t x}^{2} b-h \sin \theta \partial_{t} b\right)\left(\frac{u^{2}}{2}+g b\right)\right. \\
& \left.+g \frac{h^{3}}{3} \cos ^{3} \theta \partial_{t x}^{2} b-g \frac{h^{2}}{2} \sin \theta \cos \theta \partial_{t} b\right) \leq-\frac{u^{2}}{2} \frac{\rho_{r}}{\rho_{m}} \partial_{t} b-\left(\frac{\rho_{r}}{\rho_{m}}-1\right) g h \cos \theta \partial_{t} b
\end{aligned}
$$

which is an identity for smooth solutions,

(ii) it has the lake-at-rest solution $u=0, h \cos \theta+b=C$ st, for arbitrary given $b(t, x)$ if $\rho_{m}=\rho_{r}$, or for $b$ satisfying $\partial_{t} b \equiv 0$ if $\rho_{m} \neq \rho_{r}$,

(iii) under assumptions (3.1), (3.3), it provides in the limit $\varepsilon \rightarrow 0$ an approximation up to error terms of order $\varepsilon^{3}$ in (3.8) and of order $\varepsilon^{2}$ in (3.9), with constant velocity profile in the normal variable, to the free surface incompressible Euler equations (2.54), (2.60), (2.62), (2.72), (2.10), (2.74),

(iv) for constant slope $\left(\partial_{t} \theta=0, \partial_{x} \theta=0\right)$, system (3.8)-(3.9) gives an exact solution to the free surface incompressible Euler system with hydrostatic assumption (2.54), (2.60), (2.77), (2.72), (2.10), (2.74).

Proof. For (i), a long calculation shows that for smooth solutions, by multiplying (3.8) by $u^{2} / 2+g h \cos \theta+g b,(3.9)$ by $h u$ and adding the results, using (2.1), (2.5), we obtain exactly the equality in (3.11). Since the details of the computation are very tedious, we prefer to omit them, and rather give an abstract argument. Consider a solution $(h, u)$ to (3.8)-(3.9). Set $U(t, x, Z)=u(t, x)$, define $W$ by (2.54) with the boundary condition $(2.72)$, and take $P=g \cos \theta(h-Z)$, that satisfies the boundary condition (2.10). Then, the mass conservation (2.74) holds, and only the velocity equations for $U$ and $W$ fail to hold in order to have a solution to the Euler equations. We have the equation $\partial_{Z}(g(b+Z \cos \theta)+P)=0$, thus we can say that the hydrostatic equation (2.77) holds modulo the term $-U\left(\partial_{x}\left(\cos \theta \partial_{t} b\right)+U \partial_{x} \theta\right)$. For $U$, we can say that (2.60) holds modulo the term $W\left(\partial_{x}\left(\cos \theta \partial_{t} b\right)+U \partial_{x} \theta\right)$, with a right-hand side

$$
(h / 2-Z)\left(\partial_{x} \theta \partial_{t} u-\cos ^{2} \theta \partial_{t x}^{2} b \partial_{x} u\right),
$$


just because (3.9) has been obtained by taking $Z=h / 2$ in (2.60). Performing the same computation as for the hydrostatic model, we can derive an energy equation. We observe that the terms involving $\partial_{x}\left(\cos \theta \partial_{t} b\right)+U \partial_{x} \theta$ simplify, thus we get the energy equation (2.79) with the right-hand side $u$ times (3.12). Integrating this equation for $Z$ between 0 and $h$ then gives (2.80), since the integral in $Z$ of (3.12) vanishes. Finally the evaluation of (2.80) gives (3.11).

The property (ii) can be obtained as follows. We have to prove that if $u=0$ and $h \cos \theta+b=C$ independent of $x$ and $t$, then we have a solution to (3.8)(3.9), provided that $\left(\frac{\rho_{r}}{\rho_{m}}-1\right) \partial_{t} b \equiv 0$. The second equation (3.9) holds trivially, thus we just have to prove the first, (3.8). We first observe that $\vec{U}=0, P=$ $g C-\vec{g} \cdot \vec{X}$ gives a solution to (2.6)-(2.7). Since the free surface is defined by $h(t, x)$ such that $h \cos \theta+b=C$, it is horizontal. Thus (2.9) is satisfied. The boundary condition (2.13) holds according to the assumption, and the pressure $P$ also vanishes at the free surface, ensuring that (2.10) holds. Therefore, we have a solution to the incompressible Euler equations with boundary conditions. Formulating this solution in the $(t, x, Z)$ coordinates reads in particular as equation (2.74), proving the result. Another proof is to differentiate the relation $h \cos \theta+b=C$ with respect to $t$ and $x$, and to put the results directly in (3.8). Notice that when $\rho_{m}=\rho_{r}$ with $b$ depending on time, the lake-at-rest solution is not strictly speaking a steady state, since $h$ also depends on time. This is due to the fact that in this case, the interface is artificial and does not have a physical meaning, since the parts of the fluid above and below it are both at rest.

The property (iii) follows directly from the derivation above.

For (iv), consider a solution $(h, u)$ to (3.8)-(3.9). As in the proof of (i), let $U(t, x, Z)=u(t, x)$, define $W$ by (2.54) with the boundary condition (2.72), and take $P=g \cos \theta(h-Z)$. According to the assumptions that $\partial_{t} \theta=0$ and $\partial_{x} \theta=0$ (but without assuming that $\left.\partial_{t} b=0\right)$, the terms $\partial_{x}\left(\cos \theta \partial_{t} b\right)+U \partial_{x} \theta$ and (3.12) vanish. Thus the computations made in the proof of (i) show that we have an exact solution to the hydrostatic Euler system.

\subsection{Friction terms}

In this subsection we explain how from the incompressible system with non-isotropic stress tensor we can derive friction terms in the proposed model (3.8)-(3.9).

In the system $(2.54),(2.60),(2.62)$, the stress tensor $\mathcal{P}$ needs to be defined. Making the same scale analysis as previously performed for (2.62) to obtain (3.4), we obtain $\partial_{Z}\left(g Z \cos \theta+\mathcal{P}_{Z Z}\right)=\mathcal{O}(\varepsilon)$, provided that $\mathcal{P}_{X Z}=\mathcal{O}(\varepsilon)$. Thus,

$$
\mathcal{P}_{Z Z}=g \cos \theta(h-Z)+\mathcal{O}\left(\varepsilon^{2}\right)
$$

In order to specify $\mathcal{P}_{X X}$ it is necessary to give a constitutive law. With the purpose 
of simplicity in this work we set $\mathcal{P}_{X X}=\mathcal{P}_{Z Z}$, although the case $\mathcal{P}_{X X}=K \mathcal{P}_{Z Z}$ can be considered, $K=K_{\text {act/pass }}$ following the Savage-Hutter model [22]. The friction effects are introduced in the shear stress $\mathcal{P}_{Z X}$. Following the works of Savage-Hutter [22] and Gray [12], and in line with (2.10), we impose as boundary condition $\mathcal{P}_{Z X}$ to vanish at the free surface, and a Coulomb friction term at the flowing edge of the interface,

$$
\left(\mathcal{P}_{Z X}\right)_{\left.\right|_{Z=h}}=0, \quad\left(\mathcal{P}_{Z X}\right)_{\left.\right|_{Z=0}}=-\mu \operatorname{sgn}(U)\left(\mathcal{P}_{Z Z}\right)_{\left.\right|_{Z=0}},
$$

where $\mu=\tan \theta_{s}$ and $\theta_{s}$ is the basal friction angle. Now, if we assume that $\mu=\mathcal{O}(\varepsilon)$, we obtain $\mathcal{P}_{Z X}=\mathcal{O}\left(\varepsilon^{2}\right)$. Arguing as in the beginning of Section 3, the only new term appearing in $(2.60)$ is $\partial_{Z}\left(J \mathcal{P}_{Z X} / \cos \theta\right)$. Thus, averaging $(2.60)$ between $Z=0$ and $Z=h$, we are led to

$$
\begin{aligned}
& \left(\frac{1}{\cos \theta}-\frac{h}{2} \partial_{x} \theta\right) \partial_{t} u+\left(\frac{h}{2} \cos ^{2} \theta \partial_{t x}^{2} b-\sin \theta \partial_{t} b\right) \partial_{x} u+\partial_{x}\left(\frac{u^{2}}{2}+g h \cos \theta+g b\right) \\
& =\frac{\left.\mathcal{P}_{Z X}\right|_{Z=0}}{h \cos \theta}+\mathcal{O}\left(\varepsilon^{2}\right) .
\end{aligned}
$$

However, according to (3.14) and (3.13), we have $\left.\mathcal{P}_{Z X}\right|_{Z=0}=-\mu g h \cos \theta \operatorname{sgn}(u)+$ $\mathcal{O}\left(\varepsilon^{3}\right)$. Therefore, neglecting terms in $\varepsilon^{2}$ in $(3.15)$, we obtain together with $(2.74)$ the system

$$
\begin{gathered}
\partial_{t}\left(\frac{h}{\cos \theta}-\frac{h^{2}}{2} \partial_{x} \theta\right)+\partial_{x}\left(\frac{h^{2}}{2} \cos ^{2} \theta \partial_{t x}^{2} b-h \sin \theta \partial_{t} b+h u\right)=-\frac{\rho_{r}}{\rho_{m}} \partial_{t} b \\
\left(\frac{1}{\cos \theta}-\frac{h}{2} \partial_{x} \theta\right) \partial_{t} u+\left(\frac{h}{2} \cos ^{2} \theta \partial_{t x}^{2} b-\sin \theta \partial_{t} b\right) \partial_{x} u \\
+\partial_{x}\left(\frac{u^{2}}{2}+g h \cos \theta+g b\right)=-g \mu \operatorname{sgn}(u) .
\end{gathered}
$$

A more precise argument is indeed to say that taking into account the conditions on $\mathcal{P}_{Z X}$, a possible ansatz is

$$
\mathcal{P}_{Z X}=-\mu \operatorname{sgn}(u) g \cos \theta(h-Z)+\mathcal{O}\left(\varepsilon^{3}\right) .
$$

Then we obtain

$$
\partial_{Z}\left(\frac{J \mathcal{P}_{Z X}}{\cos \theta}\right)=g \mu \operatorname{sgn}(u)+\mathcal{O}\left(\varepsilon^{2}\right)
$$

Substituting in (2.60) gives (3.17), up to terms of order $\varepsilon^{2}$.

\subsection{Simplified model}

The model (3.8)-(3.9) is a bit complicated, thus it is tempting to try to simplify it, by dropping small terms. However, doing this, we break the nice properties stated in Theorem 3.1. However, it is worthwhile to state the result. The simplified system is defined as

$$
\partial_{t}\left(\frac{h}{\cos \theta}\right)+\partial_{x}\left(-h \sin \theta \partial_{t} b+h u\right)=-\frac{\rho_{r}}{\rho_{m}} \partial_{t} b
$$




$$
\frac{1}{\cos \theta} \partial_{t} u+\left(-\sin \theta \partial_{t} b\right) \partial_{x} u+\partial_{x}\left(\frac{u^{2}}{2}+g h \cos \theta+g b\right)=0 .
$$

The corresponding conservative momentum equation is

$$
\begin{aligned}
& \partial_{t}\left(\frac{h}{\cos \theta} u\right)+\partial_{x}\left(\left(-h \sin \theta \partial_{t} b+h u\right) u+g \frac{h^{2}}{2} \cos \theta\right) \\
& =-g \sin \theta\left(\frac{h}{\cos \theta}-\frac{h^{2}}{2} \partial_{x} \theta\right)-\frac{\rho_{r}}{\rho_{m}} u \partial_{t} b,
\end{aligned}
$$

obtained by multiplying (3.20) by $u,(3.21)$ by $h$ and adding the results.

Theorem 3.2 The system (3.20)-(3.21) has the following properties.

(i) It admits an energy dissipation inequality

$$
\begin{gathered}
\partial_{t}\left(\frac{h}{\cos \theta}\left(\frac{u^{2}}{2}+g b\right)+g \frac{h^{2}+\frac{\rho_{r}}{\rho_{m}} b^{2}}{2}\right) \\
+\partial_{x}\left(\left(\frac{u^{2}}{2}+g h \cos \theta+g b\right) h u+\left(-h \sin \theta \partial_{t} b\right)\left(\frac{u^{2}}{2}+g b\right)\right. \\
\left.-g \frac{h^{2}}{2} \sin \theta \cos \theta \partial_{t} b\right) \leq-\frac{u^{2}}{2} \frac{\rho_{r}}{\rho_{m}} \partial_{t} b-\left(\frac{\rho_{r}}{\rho_{m}}-1\right) g h \cos \theta \partial_{t} b \\
+\frac{1}{2} g h^{2}\left(\partial_{x} \theta \partial_{t} b-\partial_{x} b \partial_{t} \theta\right)
\end{gathered}
$$

which is an identity for smooth solutions,

(ii) it has the lake-at-rest solution $u=0, h \cos \theta+b=C$ st, whenever $\partial_{t} b \equiv 0$,

(iii) under assumptions (3.1), (3.3), it provides in the limit $\varepsilon \rightarrow 0$ an approximation up to error terms of order $\varepsilon^{3}$ in (3.20) and of order $\varepsilon^{2}$ in (3.21), with constant velocity profile in the normal variable, to the free surface incompressible Euler equations (2.54), (2.60), (2.62), (2.72), (2.10), (2.74),

(iv) for constant slope $\left(\partial_{t} \theta=0, \partial_{x} \theta=0\right)$, system (3.20)-(3.21) gives an exact solution to the free surface incompressible Euler system with hydrostatic assumption (2.54), (2.60), (2.77), (2.72), (2.10), (2.74).

Proof. Property (iii) follows from the fact that we only dropped terms of order $\varepsilon^{3}$ in (3.20) and $\varepsilon^{2}$ in (3.21) as compared to (3.8)-(3.9). Property (iv) is also obvious since there is no difference between (3.20)-(3.21) and (3.8)-(3.9) when $\partial_{t} \theta=0, \partial_{x} \theta=0$. For (ii), we observe that $\partial_{t} b=0$ implies that $\partial_{t} \theta=0$. Thus, differentiating the identity $h \cos \theta+b=$ Cst with respect to time yields $\partial_{t} h=0$. We deduce obviously that (3.20)-(3.21) hold. 
In order to prove (i), one multiplies (3.20) by $u^{2} / 2+g h \cos \theta+g b,(3.21)$ by $h u$ and add the results. This gives

$$
\begin{gathered}
\underbrace{g h \cos \theta \partial_{t}\left(\frac{h}{\cos \theta}\right)}_{(* 1)}+\partial_{t}\left(\frac{h}{\cos \theta} \frac{u^{2}}{2}+\frac{\rho_{r}}{\rho_{m}} \frac{g b^{2}}{2}\right)+\underbrace{g b \partial_{t}\left(\frac{h}{\cos \theta}\right)+g h \cos \theta \partial_{t} b}_{(* 2)} \\
+\underbrace{\left(\frac{u^{2}}{2}+g b\right) \partial_{x}\left(-h \sin \theta \partial_{t} b\right)-h \sin \theta \partial_{t} b \partial_{x}\left(\frac{u^{2}}{2}\right)}_{(* 3)}+\underbrace{g h \cos \theta \partial_{x}\left(-h \sin \theta \partial_{t} b\right)}_{R 1} \\
+\partial_{x}\left(\left(\frac{u^{2}}{2}+g h \cos \theta+g b\right) h u\right)=-\frac{u^{2}}{2} \frac{\rho_{r}}{\rho_{m}} \partial_{t} b-\left(\frac{\rho_{r}}{\rho_{m}}-1\right) g h \cos \theta \partial_{t} b .
\end{gathered}
$$

Then, according to $(2.1),(2.5)$, one sees that

$$
(* 1)=\partial_{t}\left(\frac{g h^{2}}{2}\right)+\underbrace{g h^{2} \sin \theta \cos \theta \partial_{t x}^{2} b}_{R 2}, \quad(* 2)=\partial_{t}\left(g b \frac{h}{\cos \theta}\right) \underbrace{-g h \sin \theta \partial_{x} b \partial_{t} b}_{(* 4)} .
$$

Moreover, we observe that

$$
(* 3)+(* 4)=\partial_{x}\left(-h \sin \theta\left(\frac{u^{2}}{2}+g b\right) \partial_{t} b\right),
$$

while a computation gives

$$
R 1+R 2=\partial_{x}\left(-g \frac{h^{2}}{2} \sin \theta \cos \theta \partial_{t} b\right)+\frac{1}{2} g h^{2}\left(\sin \theta \cos \theta \partial_{t x}^{2} b-\partial_{x} \theta \partial_{t} b\right) .
$$

Putting the results together, we obtain (3.23).

We remark that compared to the original model (3.8)-(3.9), the simplified model (3.20)-(3.21) has weaker properties (i) because there is a right-hand side proportional to $h^{2}$ (but of order $\varepsilon^{3}$ ), and (ii) because that property holds only when $\partial_{t} b=0$. We can also observe that keeping the terms involving $-\sin \theta \partial_{t} b$ in (3.20) and (3.21) is essential in order to maintain an accuracy of second order.

\subsection{Hyperbolicity}

In this subsection we study the hyperbolicity of the simplified model (3.20)-(3.21). We show that it is a hyperbolic system with eigenvalues depending of the exchange velocity between the static and the moving layers $\partial_{t} b$.

We choose the conservative variables

$$
H=\frac{h}{\cos \theta}, \quad Q=\frac{h u}{\cos \theta},
$$


and assume that $b(t, x)$ is a given smooth function. Then, according to (3.22), the system can be written as a conservative system with source term

$$
\partial_{t} V+\partial_{x}(F(t, x, V))=G(t, x, V)
$$

with $V=\left(\begin{array}{c}H \\ Q\end{array}\right)$ and

$$
\begin{gathered}
F(t, x, V)=\left(\begin{array}{c}
-H \sin \theta \cos \theta \partial_{t} b+Q \cos \theta \\
\frac{Q^{2}}{H} \cos \theta-Q \sin \theta \cos \theta \partial_{t} b+\frac{1}{2} g H^{2} \cos ^{3} \theta
\end{array}\right), \\
G(t, x, V)=\left(\begin{array}{c}
-\frac{\rho_{r}}{\rho_{m}} \partial_{t} b \\
-g H \sin \theta+g \frac{H^{2}}{2} \sin \theta \cos ^{2} \theta \partial_{x} \theta-\frac{\rho_{r}}{\rho_{m}} \frac{Q}{H} \partial_{t} b
\end{array}\right) .
\end{gathered}
$$

We can compute the eigenvalues of the jacobian matrix $\partial_{V} F$. They are given by

$$
\lambda^{ \pm}=\cos \theta\left(u-\sin \theta \partial_{t} b \pm \cos \theta \sqrt{g H}\right) .
$$

The corresponding eigenvectors are

$$
X^{ \pm}=\left(\begin{array}{c}
1 \\
u \pm \cos \theta \sqrt{g H}
\end{array}\right) .
$$

If we compare this result with the classical Saint Venant system, we see that the difference in the expression of the eigenvalues comes from the velocity $u-\sin \theta \partial_{t} b$. We observe that the term $\sin \theta \partial_{t} b$ appears because $\partial_{t} b$ is the vertical exchange velocity between the moving and the static layers (see [12]). Indeed, $\sin \theta \partial_{t} b$ is the projection of this velocity in the same direction as $u$, i.e. parallel to the interface.

\subsection{Comparison with other models}

In this subsection we compare the proposed simplified model (3.20)-(3.21) with other well-known previously proposed models. We observe that some models have a non-consistent energy equation.

At first, the model (3.20)-(3.21) deserves a comment concerning the energy balance. Indeed, one could think of a modified model

$$
\begin{gathered}
\partial_{t}\left(\frac{h}{\cos \theta}\right)+\partial_{x}\left(-h \sin \theta \partial_{t} b+h u\right)=-\frac{\rho_{r}}{\rho_{m}} \partial_{t} b \\
\frac{1}{\cos \theta} \partial_{t} u-\sin \theta \partial_{t} b \partial_{x} u+\partial_{x}\left(\frac{u^{2}}{2}+g h \cos \theta+g b\right)=(1-\delta) \frac{\rho_{r}}{\rho_{m}} \frac{u}{h} \partial_{t} b,
\end{gathered}
$$


for some parameter $\delta$ ( $\delta=1$ for the original model). Then this $\delta$-model has the associated energy equation

$$
\begin{aligned}
& \partial_{t}\left(\frac{h}{\cos \theta}\left(\frac{u^{2}}{2}+g b\right)+g \frac{h^{2}+\frac{\rho_{r}}{\rho_{m}} b^{2}}{2}\right) \\
&+\partial_{x}\left(\left(\frac{u^{2}}{2}+g h \cos \theta+g b\right) h u+\left(-h \sin \theta \partial_{t} b\right)\left(\frac{u^{2}}{2}+g b\right)\right. \\
&\left.-g \frac{h^{2}}{2} \sin \theta \cos \theta \partial_{t} b\right) \leq\left.\frac{1}{2}-\delta\right) \frac{\rho_{r}}{\rho_{m}} u^{2} \partial_{t} b-\left(\frac{\rho_{r}}{\rho_{m}}-1\right) g h \cos \theta \partial_{t} b \\
&+\frac{1}{2} g h^{2}\left(\partial_{x} \theta \partial_{t} b-\partial_{x} b \partial_{t} \theta\right) .
\end{aligned}
$$

If we use the variables $H, Q$ as in Subsection 3.5, the only difference between these models is the last term of the second component of $G$ : the term $-\frac{\rho_{r}}{\rho_{m}} u \partial_{t} b$ is replaced by $-\delta \frac{\rho_{r}}{\rho_{m}} u \partial_{t} b$. Thus the $\delta$-model can be written as (3.29), where $F$ is defined by (3.30) and

$$
G(t, x, V)=\left(\begin{array}{l}
-\partial_{t} b \\
-g H \sin \theta+g \frac{H^{2}}{2} \sin \theta \cos ^{2} \theta \partial_{x} \theta-\delta \frac{\rho_{r}}{\rho_{m}} \frac{Q}{H} \partial_{t} b
\end{array}\right) .
$$

The particular value $\delta=1 / 2$ has the property to give a conservative energy equation (3.36), up to third-order terms, independently of the choice of $b(t, x)$. We could think that this model comes from a profile of $U(t, x, Z)$ that vanishes at $Z=0$, making the corresponding term on the right-hand side of (2.80) vanish. However, we have not been able to find such a profile, solution to (2.60). Therefore, for now, only the model $\delta=1$ is justified.

\section{Differences with other models}

At first we observe that the most usual way to write erosive avalanche models is in the $t, X, Z$ variables (see for example [12], [13]). Moreover, it is generally assumed that the axis along which the $X$ variable is defined corresponds to a fixed constant angle over all the domain. Here we include the case where $\theta$ can vary in time and space. Other differences are the following.

To our knowledge, the terms involving $\sin \theta \partial_{t} b$ in (3.20), (3.21) do not appear in any other model. This has at least two consequences:

i) If a model does not take into account these terms, then it has equations valid only up to first-order, but not to second-order terms in $\varepsilon$ (or one has to assume that $\left.\partial_{t} b=\mathcal{O}\left(\varepsilon^{2}\right)\right)$. 
ii) The eigenvalues of the Jacobian matrix are only $\cos \theta(u \pm \cos \theta \sqrt{g H})$, and do not depend on the exchange velocity between the moving and the static layers $\partial_{t} b$.

Concerning the value of $\delta$, in [12], Gray deduces a model which corresponds to $\delta=1$, as in our model. However, in most other papers related to erosion in avalanches, the authors neglect the term in $\frac{Q}{H} \partial_{t} b$ in (3.37), leading to the case $\delta=0$. We have not found in the literature any model corresponding to $\delta=1 / 2$. In order to understand the influence of the value of $\delta$, we can focus on the associated energy equation. We observe that for $\delta=0$ or $\delta=1$ the right-hand side $\left(\frac{1}{2}-\delta\right) \frac{\rho_{r}}{\rho_{m}} u^{2} \partial_{t} b$ in (3.36) has either positive or negative sign according to the sign of $\partial_{t} b$. Intuitively, when $\partial_{t} b>0$ for example, part of the fluid that was moving comes to rest. Thus the effect of $\partial_{t} b>0$ is to remove some kinetic energy, leading to a negative right-hand side in the energy equation. This indicates that the value $\delta=0$ is not compatible with reasonable energy considerations. Another argument is to observe that since the model is related to integrals of the solution to the Euler system, the associated energy equation must be consistent with the integrated energy equation of the Euler system. Comparing the right-hand side $-\frac{U(Z=0)^{2}}{2} \frac{\rho_{r}}{\rho_{m}} \partial_{t} b$ in the integrated energy equation $(2.80)$ to $\left(\frac{1}{2}-\delta\right) \frac{\rho_{r}}{\rho_{m}} u^{2} \partial_{t} b$, we conclude that only the value $\delta=1$ is consistent if $U(Z=0)=u$. With $\delta=0$ we get $\frac{1}{2} \frac{\rho_{r}}{\rho_{m}} u^{2} \partial_{t} b$, which cannot be obtained with any definition of $U(Z=0)$ in $(2.80)$.

\section{Models with small velocity and equation for $b$}

In this section we show how it is possible to relate our model to previously proposed systems that are closed with an equation for $b$. These models assume a small velocity.

\subsection{Linear velocity profile}

In [2], [9] and [13], the authors obtain an equation that describes the evolution of $b$ in the case of a linear profile of the velocity, $U(t, x, Z)=U_{1}(t, x) Z+\mathcal{O}\left(\varepsilon^{2}\right)$. Since $U_{1}$ is assumed to be bounded, this implies that $U=\mathcal{O}(\varepsilon)$. The linearity assumption is related to the assumption $U(Z=0)=0$, which seems reasonable when friction occurs at the interface (but not satisfactory according to K. Hutter). The model with friction, as described in Subsection 3.3, is considered here. The profile of $U$ is introduced in equation (2.60) and the terms corresponding to different powers of $Z$ are put together. To close the equation, a parametrization of the value of $\partial_{Z}\left(\mathcal{P}_{Z X} / \cos \theta\right)$ is needed. Let us consider a linear or parabolic profile for the shear stress

$$
\mathcal{P}_{Z X}=C+A Z+B \frac{Z^{2}}{2}+\mathcal{O}\left(\varepsilon^{3}\right)
$$


Then, substituting (4.1) into (2.60) and grouping together the terms that are constant and linear in $Z$, respectively, we deduce the following two equations,

$$
\begin{gathered}
\partial_{x}(g h \cos \theta+g b)-U_{1} \partial_{t} b=\frac{-A}{\cos \theta}+\mathcal{O}\left(\varepsilon^{2}\right), \\
\frac{1}{\cos \theta} \partial_{t} U_{1}-\sin \theta \partial_{t} b \partial_{x} U_{1}=\frac{-B}{\cos \theta}+\mathcal{O}(\varepsilon) .
\end{gathered}
$$

If we consider a linear profile of $\mathcal{P}_{Z X}$ (i.e. $B=0$ ), the boundary conditions $(3.14)$ give

$$
C=-\mu \operatorname{sgn}(U)\left(\mathcal{P}_{Z Z}\right)_{\left.\right|_{Z=0}}, \quad A=\frac{\mu \operatorname{sgn}(U)\left(\mathcal{P}_{Z Z}\right)_{\left.\right|_{Z=0}}}{h}
$$

Moreover, recalling that $\partial_{x} \theta=\mathcal{O}(\varepsilon)$ and $\partial_{t} b=\mathcal{O}(\epsilon)$ and neglecting second-order terms (if $U_{1}$ is bounded), we obtain the system

$$
\begin{gathered}
\partial_{x}(g h \cos \theta+g b)-U_{1} \partial_{t} b=-g \mu \operatorname{sgn}\left(U_{1}\right), \\
\partial_{t} U_{1}=0 .
\end{gathered}
$$

It is completed by the mass conservation equation obtained from (2.74),

$$
\partial_{t}\left(\frac{h}{\cos \theta}-\frac{h^{2}}{2} \partial_{x} \theta\right)+\partial_{x}\left(\frac{h^{2}}{2} \cos ^{2} \theta \partial_{t x}^{2} b-h \sin \theta \partial_{t} b+\frac{1}{2} h^{2} U_{1}\right)=-\frac{\rho_{r}}{\rho_{m}} \partial_{t} b .
$$

We observe that the system (4.5)-(4.7) has the lake at rest solution

$$
U_{1}=0, \quad b+h \cos \theta=\text { Cst }
$$

for arbitrary $b(t, x)$ if $\rho_{r}=\rho_{m}$, or for $\partial_{t} b=0$ otherwise.

\subsection{Khakhar's model}

Khakhar et al. in [13] propose an equation for the change of $b$ with time. In his model the effect of pressure gradient is neglected. Thus, in order to compare their with our model, we neglect first-order terms in (4.5). Keeping in mind that $\partial_{x} b=\tan \theta$ and $\mu=\tan \theta_{s}$, we obtain from (4.5)

$$
\partial_{t} b=\frac{g}{U_{1}}\left(\tan \theta+\operatorname{sgn}\left(U_{1}\right) \tan \theta_{s}\right)
$$

If we consider that $0 \leq \theta<\pi / 2$ then $\operatorname{sgn}\left(U_{1}\right)=-1$ and we obtain

$$
\partial_{t} b=\frac{g}{U_{1}}\left(\tan \theta-\tan \theta_{s}\right)=\frac{g \sin \left(\theta-\theta_{s}\right)}{U_{1} \cos \theta \cos \theta_{s}} .
$$


However, we do not have any expression for $U_{1}$. Khakhar et al. [13] study this quasi-stationary case, and they obtain an expression for $U_{1}$,

$$
U_{1}= \pm \sqrt{\frac{g \cos \theta \sin \left(\theta_{m}-\theta_{s}\right)}{c d \cos \theta_{m} \cos \theta_{s}}}
$$

This expression is deduced from the assumptions on the friction term. Following experimental results, the friction term in the flowing layer is assumed to be the sum of a Bagnold term and a Coulomb term,

$$
\left(\mathcal{P}_{Z X}\right)_{\left.\right|_{Z=0}}=-\operatorname{sgn}(U)\left(c d h\left(\frac{\partial U}{\partial Z}\right)^{2}+g h \cos \theta \tan \theta_{s}\right),
$$

where $c \approx 1.5$ is a parameter of the model, $d$ is the diameter of the particles and $\theta_{s}$ is the static angle of repose (for more details see [13]). They identify this expression with the stress of the flowing particles in the upper layer, which is supposed to be

$$
\left(\mathcal{P}_{Z X}\right)_{\left.\right|_{Z=0}}=g(h+d) \cos \theta \tan \theta_{m}
$$

where $\theta_{m}$, as described by Khakhar et al. in [13], is the "maximum angle of repose". Indeed, $\tan \theta_{s}$ is the effective coefficient of dynamic friction and $\tan \theta_{m}$ the effective coefficient of static friction. From (4.12) and (4.13), equation (4.11) can be deduced.

With the above definition of the velocity profile, Khakhar et al. study the quasistationary case, and deduce the following equation for $b$,

$$
\partial_{t} b=\frac{g}{U_{1}}\left(\tan \theta-\tan \theta_{m}\right)
$$

If we compare this with equation (4.10) obtained for $b$ in our model, $\theta_{m}$ is replaced by $\theta_{s}$. However, we have only considered a Coulomb friction term. If we consider $\mathcal{P}_{Z X}$ defined by (4.12) we also obtain (4.14).

\subsection{The BCRE model}

In [2] the Saint Venant model is compared with the BCRE phenomenological model. The BCRE model reflects the exchange of mass and the advection of particles in the upper layer. As described in the introduction, the BCRE model reads

$$
\left\{\begin{array}{l}
\partial_{t}\left(\frac{h}{\cos \theta}\right)+\partial_{x}\left(h \mathcal{V}_{d}\right)=\mathcal{E}(t, x) \\
\partial_{t} b=-\mathcal{E}(t, x)
\end{array}\right.
$$

with $\mathcal{E}$ the mass exchange between the flowing and static layer. In this subsection we consider $\theta$ to be almost constant, and we assume that $\rho_{m}=\rho_{r}$. By $\mathcal{V}_{d}$ we denote 
the velocity of grains in the upper layer (see [2], [7], [8]). Owing to equation (1.4), the BCRE model can be written as

$$
\left\{\begin{array}{l}
\partial_{t} b=-\mathcal{V}_{u p}\left(\theta-\theta_{n}\right) \\
\partial_{t}\left(\frac{h}{\cos \theta}\right)+\partial_{x}\left(h \mathcal{V}_{d}\right)=\mathcal{V}_{u p}\left(\theta-\theta_{n}\right)
\end{array}\right.
$$

where $\mathcal{V}_{u p}\left(\theta-\theta_{n}\right)$ is the exchange term between the static and the mobile layers. By $\theta_{n}$ one denotes a neutral angle, which separates the erosion and deposition profile. The factor $\mathcal{V}_{u p}$ has the dimension of a velocity and is a parameter of the model. For more details see [2], [7], [8]. In [2], Aradian et al. relate this model to the Saint Venant model to derive the parameters $\mathcal{V}_{u p}$ and $\mathcal{V}_{d}$ involved in the BCRE model. Nevertheless, as usual, they neglect the term $U_{l_{b}} \partial_{t} b$ in the momentum equation. We show here that including this term can change the determination of the parameter $\mathcal{V}_{u p}$ by a factor $3 / 2$.

Let us start as in the derivation of the friction proposed in Subsection 3.3, but instead of a constant velocity profile, we rather take, as in [2],

$$
U=-\Gamma_{0} Z
$$

where $\Gamma_{0}=\sqrt{g / d}$ and $d$ is the grain diameter. Thus $U<0$ which is coherent with the situation, when $0 \leq \theta<\pi / 2$. Observe that (4.17) gives $\partial_{t} U=0, \partial_{x} U=0$. Then, since $u \equiv \bar{U}=-\Gamma_{0} h / 2$, averaging (2.60) between $Z=0$ and $Z=h$ gives

$$
\partial_{x}(g h \cos \theta+g b)+\Gamma_{0} \partial_{t} b=\frac{\left.\mathcal{P}_{Z X}\right|_{Z=0}}{h \cos \theta}+(1-\delta) \frac{u}{h} \partial_{t} b+\mathcal{O}\left(\varepsilon^{2}\right),
$$

with

$$
\left.\mathcal{P}_{Z X}\right|_{Z=0}=-\mu \operatorname{sgn}(U) g h \cos \theta
$$

In the above, $\delta$ is a parameter, formally inserted to identify (4.18) with (3.35). This has to be completed by the mass conservation equation obtained from (2.74),

$$
\partial_{t}\left(\frac{h}{\cos \theta}-\frac{h^{2}}{2} \partial_{x} \theta\right)+\partial_{x}\left(\frac{h^{2}}{2} \cos ^{2} \theta \partial_{t x}^{2} b-h \sin \theta \partial_{t} b-\Gamma_{0} \frac{h^{2}}{2}\right)=-\partial_{t} b .
$$

Neglecting second-order terms gives

$$
\partial_{t}\left(\frac{h}{\cos \theta}\right)+\partial_{x}\left(-\Gamma_{0} \frac{h^{2}}{2}\right)=-\partial_{t} b+\mathcal{O}\left(\varepsilon^{2}\right) .
$$

Combining (4.21) and (4.18) where we neglect the term $\partial_{x}(g h \cos \theta)$, we obtain

$$
\left\{\begin{array}{l}
\partial_{t}\left(\frac{h}{\cos \theta}\right)+\partial_{x}\left(-\Gamma_{0} \frac{h^{2}}{2}\right)=\frac{g}{\Gamma_{0} \cos \theta(3-\delta) / 2}(\sin \theta-\mu \cos \theta) \\
\partial_{t} b=\frac{-g}{\Gamma_{0} \cos \theta(3-\delta) / 2}(\sin \theta-\mu \cos \theta)
\end{array}\right.
$$


If $\mu=\tan \theta_{s}$ then

$$
\sin \theta-\mu \cos \theta=\frac{1}{\cos \theta_{s}} \sin \left(\theta-\theta_{s}\right) .
$$

Then, if $\theta \approx \theta_{s}$ the following system is obtained,

$$
\left\{\begin{array}{l}
\partial_{t}\left(\frac{h}{\cos \theta}\right)+\partial_{x}\left(-\Gamma_{0} \frac{h^{2}}{2}\right)=\frac{g}{\Gamma_{0} \cos ^{2} \theta_{s}(3-\delta) / 2}\left(\theta-\theta_{s}\right), \\
\partial_{t} b=\frac{-g}{\Gamma_{0} \cos ^{2} \theta_{s}(3-\delta) / 2}\left(\theta-\theta_{s}\right) .
\end{array}\right.
$$

If we compare (4.16) and (4.24), following [2], we obtain the identifications

$$
\mathcal{V}_{d}=-\Gamma_{0} \frac{h}{2}, \quad \theta_{n}=\theta_{s}, \quad \mathcal{V}_{u p}=\frac{g}{\Gamma_{0} \cos ^{2} \theta_{s}(3-\delta) / 2}
$$

We observe that since $u=-\Gamma_{0} h / 2$, we have $\mathcal{V}_{d}=u$. Finally, we observe that $\mathcal{V}_{u p}$, the uphill velocity of exchange from the static to the mobile layers, is modified by the choice of $\delta$. In fact, one has $\left(\mathcal{V}_{u p}\right)_{\delta=1}=\frac{3}{2}\left(\mathcal{V}_{u p}\right)_{\delta=0}$.

\section{Acknowledgments}

The authors are very grateful to

Kolumban Hutter for his detailed comments that helped a lot in improving the paper. In particular, the generalization to take into account the case when $\rho_{m} \neq \rho_{r}$ has been performed according to his suggestion.

We thank the members of GDR MIDI for fruitful discussions.

This work has been partially supported by the ACI NIM contract no 03318, the ANR contract no ANR-06-BLAN-0414, the ACI Jeunes Chercheurs "Géomorphogénèse, Erosion et Transport Granulaire", the Spanish Government Research project MTM2006-08075. IPGP contribution number 2292.

\section{References}

[1] A. Aradian, Quelques problèmes d'interface mole. Ph.D. Thesis Paris VI (2001).

[2] A. Aradian, E. Raphael, P.-G. de Gennes, Surface flow of granular materials: a short introduction to some recent models. C.R. Physique 3, 187-196 (2002).

[3] I.S. Aranson, L.S. Tsimring, Continuum theory of partially fluidized granular flows. Phys. Rev. E. 65, 061303 (2002).

[4] A. Bon, Avalanche d'un milieu granulaire. Master 2, University Paris 6.

[5] F. Bouchut, A. Mangeney-Castelnau, B. Perthame, J.-P. Vilotte, A new model of Saint Venant and Savage-Hutter type for gravity driven shallow water flows. C.R. Acad. Sci. Paris, Ser I 336, 531-536 (2003). 
[6] F. Bouchut, M. Westdickenberg, Gravity driven shallow water model for arbitrary topography. Comm. Math. Sci. 2(3), 359-389 (2004).

[7] J.P. Bouchaud, M.E. Cates, J.R. Prakash, S.F. Edwards. A model for the dynamics of sandpile surface. J. Phys. Paris I 4, 1383-1410 (1994).

[8] T. Boutreux, E. Raphael, P.G. DeGennes, Surface flows of granular materials: a modified picture for thick avalanches. Phys. Rev. E 58, 4692-4700 (1998).

[9] S. Douady, B. Andreotti, A. Daerr, On granular surface flow equations. Eur. Phys. J.B. 11, 131-142 (1999).

[10] M. Papa, S. Egashira, T. Itoh, Critical conditions of bed sediment entrainment due to drebis flow, Natural Hazards and Earth System Sciences 4, 469-474 (2004).

[11] J.-F. Gerbeau, B. Perthame, Derivation of viscous Saint Venant system for laminar shallow water; numerical validation. DCDS(B) 1 (1) (2001).

[12] J.M.N.T. Gray, Granular flow in partially filled slowly rotating drums. J. Fluid Mech 441, 1-29 (2001).

[13] D.V. Khakhar, A.V. Orpe, P. Andresén, J.M. Ottino, Surface flow of granular materials: model and experiments in heap formation. J. Fluid Mech. 441, 225264 (2001).

[14] D.V. Khakhar, A.V. Orpe, Surface granular flows: two related examples. Advances in Complex Systems 4(4), 407-417 (2001).

[15] K.K.J. Kouakou, Instabilité des interfaces écoulement/fond érodable : approches asymptotiques. Preprint University of Paris VI (2005).

[16] L.D. Landau, and Lifshitz, E.M., Statistical Physics, Pargamon Press, NewYork (1980)

[17] A. Mangeney-Castelnau, J.-P. Vilotte, M.-O. Bristeau, B. Perthame, F. Bouchut, C. Simeoni, S. Yerneni, Numerical modeling of avalanches based on Saint Venant equations using a kinetic scheme. J. Geophys. Res. 108 (B11), 2527 (2003).

[18] A. Mangeney-Castelnau, F. Bouchut, J.-P. Vilotte, E. Lajeunesse, A. Aubertin, M. Pirulli, On the use of Saint Venant equations to simulate the spreading of a granular mass, J. Geophys. Res. 110 (B9), B09103 (2005).

[19] A. Mangeney, F. Bouchut, N. Thomas, J.-P. Vilotte, M.-O. Bristeau, Numerical modeling of self-channeling granular flows and of their levee/channel deposits, to appear in J. Geophys. Res. (2007). 
[20] A. Mangeney, L.S. Tsimring, D. Volfson, I.S. Aranson, F. Bouchut, Avalanche mobility induced by the presence of an erodible bed and associated entrainment, Geophys. Res. Lett., in press (2007).

[21] Pudasaini, S., and Hutter, K., Avalanche Dynamics, Springer-Verlag, 602p (2007).

[22] S.B. Savage, K. Hutter, The dynamics of avalanches of granular materials from initiation to run-out, Acta Mech. 86, 201-223 (1991).

[23] B. Sovilla, P. Burlando, and P. Bartelt, Field experiments and numerical modeling of mass entrainment in snow avalanches J. Geophys. Res., 111, F03007 (2006).

[24] M. Wieland, J.M.N.T. Gray, K. Hutter, Channelized free-surface flow of cohesionless granular avalanches in a chute with shallow lateral curvature. J. Fluid Mech. 392, 73-100 (1999). 